\title{
How does academia respond to the burden of infectious and parasitic disease?
}

\author{
Wenjing Zhao ${ }^{1,2}$, Lili Wang ${ }^{3}$, Lin Zhang ${ }^{1,2,4 *}$ \\ ${ }^{1}$ School of Information Management, Wuhan University, Wuhan, China \\ ${ }^{2}$ Research Center for Science, Technology \& Education Management and Evaluation, Wuhan University, China \\ ${ }^{3}$ UNU-MERIT, Maastricht University, the Netherlands \\ ${ }^{4}$ Centre for R\&D Monitoring (ECOOM) and Department of MSI, KU Leuven, Leuven, Belgium
}

\begin{abstract}
:
Background: Academic research is one of the main avenues through which humans can fight the threat of infectious diseases. However, there has been concerns regarding whether academic system has provided sufficient efforts to fight infectious diseases we potentially face. Answering these questions could contribute to evidence-based recommendations for setting research priorities and third-mission policies.

Methods: With a focus on one of the most common categories of communicable diseases, infectious and parasitic diseases (IPDs), we searched Web of Science for articles and reviews relevant to IPDs published during 2000-2019 and acquired WHO data of disease burden in corresponding years. The academic response patterns were explored by IPD subcategory, and by human development level (an index established by the UN). We in particular conduct the analysis to gain insight on the dynamic relationship between disease burden and research effort on IPD, scientific efforts contributed by countries with different development levels, and the variation trends in international joint efforts.

Results: The greatest burden of IPD disease is clustered in the developing regions of Africa, but has received academic response from both developed and developing countries. Highly developed countries dominate the ranks of academic research in this area, yet there is also a clear increase of research efforts from the countries most affected, despite their low human development scale. In fact, the overall analysis reveals an improved capability of addressing local problems from African regions. In terms of international collaboration, highly developed countries, such as the US and UK, have commonly collaborated with needy regions, whereas prolific but developing nations, like China, have not.

Conclusions: From a global perspective, academia has positively responded to health need caused by IPD. Although the relevant research output is primarily contributed by the highly developed countries, the concentrated efforts from the undeveloped regions to ease their local burdens can be clearly observed. Our findings also indicate the tendency on focusing more on local health needs for both developed and undeveloped regions. The insights revealed in this study should benefit a more informed and systemic plan of research priorities.
\end{abstract}

Keywords: infectious and parasitic diseases (IPDs), academic research, response patterns, disease burden, DALYs

*Correspondence: linzhang1117@whu.edu.cn 


\section{Introduction}

Infectious diseases have, for centuries, been one of the leading causes of death and disability and, despite all human endeavor, they still present a growing challenge to health and social progress $(1,2)$. Most recently, the COVID-19 pandemic has shaken up global structures in a range of ways, leaving in its wake an urgent health need that has required rapid and innovative research to control. Biomedical research, with its principles of better patient treatment and illness prevention (3), has been regarded as a core approach to fighting not just COVID-19 but any infectious disease that would threaten our safety. And what the COVID-19 crisis has shown us is that academia can, and will, "turn on a dime" to respond to emergent health needs. As evidence of academia's adroit response, Zhang et al. (4) point to marked increases in the number of publications, while both Fry et al. (5) and Lee and Haupt (6) acknowledge changing patterns in international collaborations. However, beyond these observations of academia's tremendous response to COVID-19 and similar diseases of "Public Health Emergency of International Concerns (PHEICs)" as defined by the World Health Organization (WHO), patterns of research into disease burdens caused by commonly-existed and long-standing infectious disease is an area that warrants further research. Such research could contribute to evidence-based recommendations for setting research priorities or third-mission ${ }^{1}$ policies that help to mitigate some of the negative impacts of infectious diseases.

Long-standing infectious diseases are those that have existed for a long time in human history but do not constantly emerge as outbreaks, epidemics, or pandemics. They span many different conditions and presentations, from HIV to nematode infestations to hepatitis and leprosy. We examine 11 major subcategories of infectious and parasitic diseases in this study, collectively referring to them as IPDs. Although the burden of IPDs has decreased over the past few decades (2), the mortality rate has remained intolerably high. According to the WHO (7), IPDs killed more than 5 million people in 2019 - mostly in low- and middle-income countries. With the potential to improve the quality of life by identifying treatment regimens, biomedical research can play a vital role in combating infectious diseases that affect impoverished populations (8). Efficient and sufficient efforts from academic community are required to respond to such health challenges. Indeed, comprehensively understanding the features of an academic response to health issues is extremely difficult because research interests and priorities are shaped by many interacting social, economic, and political factors. However, a systematic study on dynamic relationship between disease burden and research effort on IPD, the scientific efforts contributed by countries with different development levels, and the variation trends in international joint efforts is an insightful exercise and useful source for expeditious and efficient strategic research plans. In this study, we adopt the Human Development Index (HDI) developed by the United Nations Development Programme (9) to classify the human development level into four groups. This index consists of factors such as life expectancy, education and per capita income and provides insight into the potential that could be achieved by a country if there were no inequality (10).

The extent to which researchers have responded to the health needs caused by infectious diseases reveals the connections between the "supply side " of science and knowledge and "demand side" in health fields

\footnotetext{
${ }^{1}$ The third mission refers to contributions of universities to the society.
} 
(11). Academic research is a complex, collaborative, and goal-oriented activity (12), driven by diverse individual and social factors (13). At the individual end of the spectrum, we have, for example, the curiosity of the researcher (14). At the societal end, we have pressure to solve the particular problems society deems important (15). However, what needs to be emphasized is that science itself is not a closed and autarkic system. Its development relies greatly on the provision of resources from other parts of society. As such, academic research is naturally expected to produce knowledge that addresses particular needs of the society (16). More and more, the huge impetus and benefits to social development science brings are being recognized. Accordingly, science policy is gradually bending toward finding answers for both pressing local issues and the world's grand challenges (17). Consequently, the relationship between science and society is changing (16) from one where "mad geniuses" are largely left to pursue their own interests to another where "educated technicians" are increasingly being asked to make substantial and specific contributions to society's needs. In other words, although the trajectory of science and technology is influenced by various factors $(15,18,19)$, a basic consensus has been reached where research priorities are, to a certain extent, set according to society's needs (20-22).

Indeed, assessing the research effort required to address complex global challenges has been drawing increasing attention from scholars in many fields - environment and energy science (23), agriculture (15) and health (24-26) to name a few. In disciplines related to health, such as biomedicine, there is an especially high expectation to focus on the diseases causing the most burden $(17,27)$. Appropriate research outcomes include improving healthcare capabilities, providing valuable information on diseases trends, insights into patterns of care, effective treatments, of course, and so on (28). Even though each type of such research contribution is affected by a vast range of factors, exploring the long-term correlations between the research effort and disease burden is a first step in broadening our understanding of this interplay, which can lead to a more informed and systemic plan of research priorities.

As metrics that reflect research effort, prior studies have considered academic research output $(29,30)$, funding $(17,21,31)$, and clinical trials $(32,33)$. The most common among these is research output, which is what we have used for this study. Due to the heterogeneous nature, it is important to focus on particular region(s) and/or disease(s), such as medical research in Africa (30), India (29) or Palestine (34), or specific chronic respiratory diseases in Europe (35), diabetes in five small countries in Europe (36), neglected tropical diseases in Brazil (37) or cancer research in 29 countries (24) or just in China (25). At the global level, Yegros-Yegros et al. (38) use research output indexed by MEDLINE and WHO data from the Global Burden of Disease in 2010 to explore the relationships between research effort and health needs for 134 diseases. The authors conclude that research output is heavily concentrated in high-income countries and is mainly focused on their own health needs. As a result, there is a relative lack of attention to diseases in lower-income countries. Similar results are also reported from analyses conducted by Hagenaars et al. (27). Here, the data indicated an uneven distribution of scientific advances across societal sectors and their diverse demands.

It is well acknowledged that health research resource is unequally distributed. Evans et al. (26) find that many of the health needs associated with infectious diseases that predominantly affect poor populations do not attract attention from the researchers in developed countries. This paper raise the concern that, in general, researchers are not devoting relatively equal effort to the needs of the "poor populations deficient in their own research infrastructure" (26). In line with this, other studies also point out the need for a 
better fit between research effort and global burden (39-41). These arguments typically rest on the long tradition of medical research - to reduce health inequality and ultimately realize universal well-being (26). Like Evans and colleagues, these scholars advocate that those rich countries should invest in R\&D that address the specific health problems of poor populations, due to the vast discrepancy between disease burden and research capacity in low-to-middle-income countries $(40,41)$. In contrast to this, another group of scholars insist that local researchers should address local problems $(21,42)$, and emphasize the need to justify public spending on health research to taxpayers (43). Yet, despite all this debate, few studies have comprehensively examined where and how the academic community exerts their research effort in terms of disease burden on a global scale. In particular, no study has done so with IPDs, which tend to have a low disease burden in high-income countries and a very high disease burden in the lowestincome countries.

The other dimension through which we analyze response patterns is academic collaboration. This element of research is of great significance in that it presents how scholars from different countries with varying backgrounds jointly defend against diseases. Several studies have been conducted to investigate the features of collaborative medical research between countries. For instance, Kozma and Calero-Medina (44) examine the role of South African scholars in intercontinental scientific collaborations in Tropical Medicine, Immunology and other relevant fields, while Zacca-González et al. (45) look at international collaborations and productivity in Latin America. However, few studies have integrated information on disease burdens into their analysis. Thus, questions over whether countries collaborate to address diseases of burden deserve investigation - especially with respect to high-burden diseases in underdeveloped regions.

In short, this research give rise to the following research questions:

1. What is the dynamic relationship between research effort and the burden of IPDs?

2. What roles do countries at various levels of human development play in contributing to scientific research related to IPD?

3. How scholars from countries at various levels of human development jointly defended against IPDs?

The rest of this paper proceeds as follows. The next section presents the data, methods, and tools used in this analysis, including a primer on IPD classifications and the sources of data used to explore IPD research. The main results and our analysis are provided in the third section. The last section contains the conclusion and discussion of results, including the limitations and reflections of future work.

\section{Data and methodology}

Figure 1 depicts the framework used to conduct this research. Each of the procedures and analysis criteria are described in this section. 


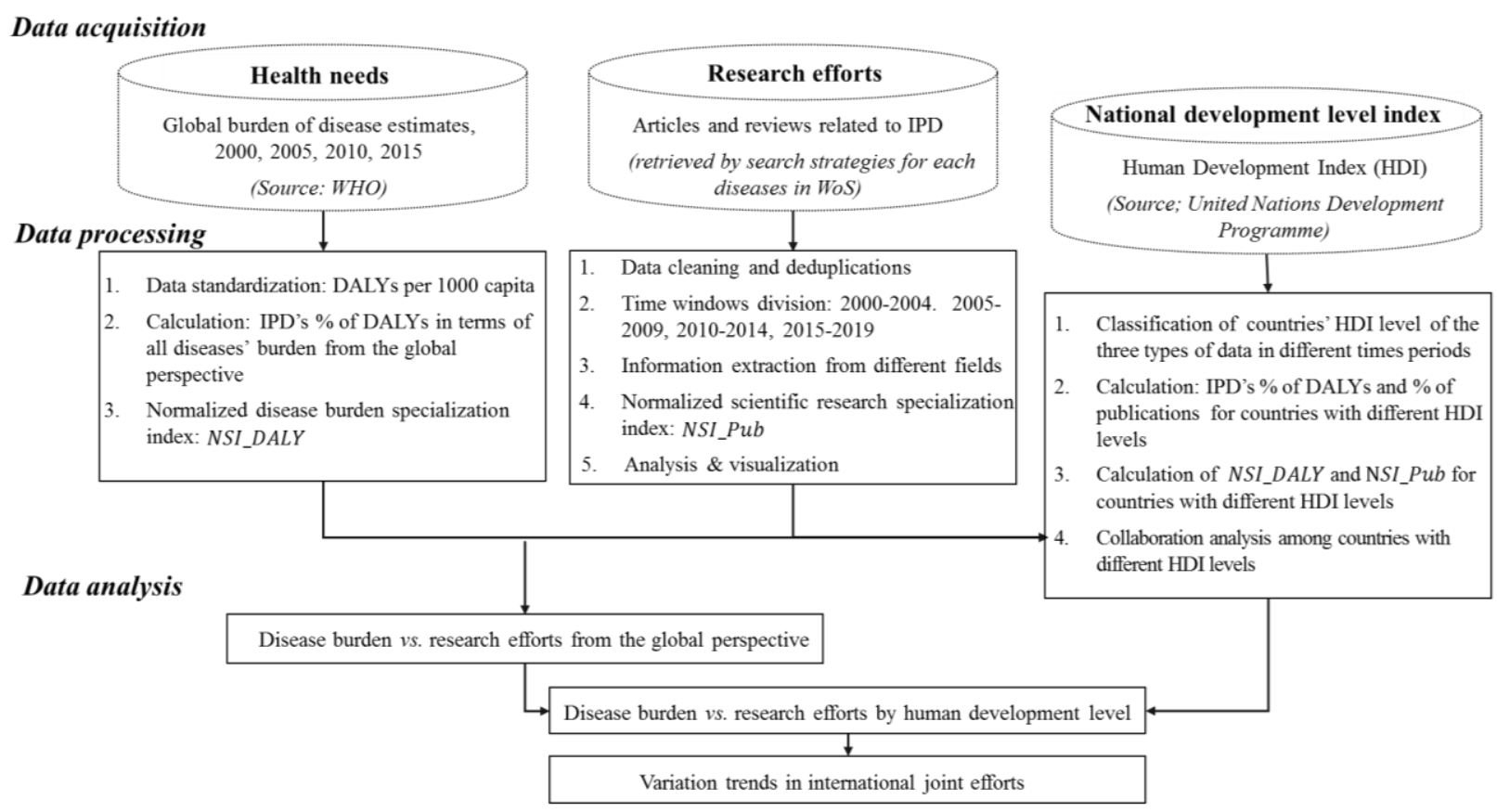

Figure 1. Research framework

\subsection{Health needs}

\subsubsection{Classification of diseases}

The Global Health Estimates (GHE) cause categories established according to International Classification of Diseases, Tenth Revision (ICD-10) by WHO (46) publishes 11 different categories of IPDs, which, combined, cover the largest aggregation of communicable diseases. These are listed in Table 1 alongside a code we will use to refer to each condition in all subsequent tables and figures.

Table 1. 11 specific conditions of infectious and parasitic diseases

\begin{tabular}{ll}
\hline Code & $\begin{array}{l}\text { Infectious and parasitic } \\
\text { diseases }\end{array}$ \\
\hline 1 & Tuberculosis \\
2 & STDs excluding HIV \\
3 & HIV/AIDS \\
4 & Diarrheal diseases \\
5 & Childhood-cluster diseases \\
6 & Meningitis \\
7 & Encephalitis \\
8 & Hepatitis \\
9 & Parasitic and vector diseases \\
10 & Intestinal nematode infections \\
11 & Leprosy \\
\hline
\end{tabular}




\subsubsection{Burden of disease}

Reliable and transparent health statistics are of great importance to anyone concerned with public health policy. To this end, a wide range of indicators have been developed to monitor and manage health initiatives. In line with similar prior studies $(17,21,26)$, we take disability-adjusted life years (DALYs) to represent the burden of disease. DALYs are essentially a sum of the time lost through premature death plus time lived in a state of less-than-optimal health. In this sense, they quantify the difference between a person's existing health status and ideal health conditions where one can expect to live to an advanced age, free of disease and disability (46).

To calculate the trends relevant to DALYs for different time stages, the DALY estimates were collected for all categories of diseases from the WHO for 183 countries in the years 2000, 2005, 2010 and 2015 (see Table 2). We then determine the burden of disease in terms of $\%$ of total DALYs for each country and each of the 11 conditions. To correct for differences in population size, we standardize the results as DALYs per 1000 capita. In addition, we adopt "disease burden specialization index (SI)" from Confraria and Wang's (30) to reflect "relative level of burden formulation in terms of the world-average level", which is also free from the impact of population. This index can be expressed as:

$$
S I_{-} D A L Y_{r d}=\frac{D_{r d} / \sum_{d} D_{r d}}{D_{d} / \sum_{d} D_{d}}
$$

where $\boldsymbol{D}_{\boldsymbol{r} \boldsymbol{d}}$ is the number of DALYs in region $\boldsymbol{r}$ for disease $\boldsymbol{d}$, and $\boldsymbol{D}_{\boldsymbol{d}}$ is the WHO's estimated DALYs resulting from the disease $\boldsymbol{d}$ worldwide. $S I_{-} D A L Y>1$ indicates that region $\boldsymbol{r}$ carries a relatively higher burden from disease $d$ than the world average, while $S I_{-} D A L Y<1$ indicates that this region carries a relatively lower burden from disease $d$ than the world average. The value range of $S I_{-} D A L Y$ is null or positive infinity. Hence, this indicator is further standardized as "normalized specialization index (NSI)" with the following approach:

$$
N S I+D A L Y=\frac{(S I D D A L Y-1)}{(S I+D A L Y+1)}
$$

Now, this measure has the asymptotic limits of \pm 1 , and the threshold value of zero.

\subsection{Research effort}

Among the various types of academic research outputs have, such as academic publications, patents, reports, etc., peer-review journals are the most common outlets for scholars to communicate about issues related to health (47). Hence, academic articles have become the standard indicator by which research effort is measured when it comes to IPDs $(38,48)$.

We retrieved our corpus of articles from Clarivate Analytics' Web of Science (WoS) Core Collection using a search strategy built by Confraria and Wang (30). The strategy comprises a set of keywords strongly associated with different categories diseases and 11 conditions of IPDs covered in this study. A more detailed explanation of search strategy with the introduction of cause category is provided in Appendices A1. We searched the title and keywords fields of WoS with a published date range of 2000 to 2019 and subsequently divided the results into four time periods of five years each (2000-04, 200509, 2010-14, and 2015-19 to be clear). In total, we retrieved 393,716 articles and reviews. Note, however, that the sum of the 11 subcategories will be more than this total since some articles belong to more than 
one disease category.

We further did a search for all disease-related publications in the same period with a view to comparing the proportion of research effort devoted to IPDs versus the total burden of disease. In total, we retrieved 2,387,505 articles from this search. To calculate the research effort, we again turned to Confraria and Wang (30), using their relative "specialization index (SI)", expressed as follows:

$$
S I_{-} P u b_{r d}=\frac{P_{r d} / \sum_{d} P_{r d}}{P_{d} / \sum_{d} P_{d}}
$$

where $\boldsymbol{P}_{\boldsymbol{r} \boldsymbol{d}}$ is the number of articles produced in region $\boldsymbol{r}$ for disease $\boldsymbol{d}$, and $\boldsymbol{P}_{\boldsymbol{d}}$ is the number of articles related to disease $\boldsymbol{d}$ published worldwide. $S I_{-} P u b>1$ indicates that region $\boldsymbol{r}$ has exerted a relatively higher research effort to address disease $d$ than the world average, SI_Pub $>1$ indicates that this region has exerted a relatively lower research effort to address disease $d$ than the world average. Similar approach as the NSI of disease burden has been also applied to normalize the SI_Pub:

$$
N S I_{-} P u b=\frac{\left(S I_{-} P u b-1\right)}{\left(S I_{-} P u b+1\right)}
$$

\subsection{The Human Development Index}

The Human Development Index (HDI) introduced by United Nations Development Programme (UNDP) serves as a more comprehensive measure of human development than purely economic measures (9). It is meant to be "a summary measure of average achievement in key dimensions of human development: a long and healthy life, being knowledgeable and having a decent standard of living." The UNDP's Data Center describes the measure as "the geometric mean of normalized indices for each of the three dimensions" - health, education, and income (49). The explanations are as follows:

The health dimension is assessed by life expectancy at birth, the education dimension is measured by mean of years of schooling for adults aged 25 years and more and expected years of schooling for children of school entering age. The standard of living dimension is measured by gross national income per capita. The HDI uses the logarithm of income, to reflect the diminishing importance of income with increasing GNI. The scores for the three HDI dimension indices are then aggregated into a composite index using geometric mean.

Based on the calculated HDI result, a country can fall into one of four categories - very high, high, medium, and low. HDIs for each country are updated every year, with the exception of North Korea and Somalia who do not have HDI indicators. Thus, we conducted our analysis on 181 countries, taking the HDIs from the years 2000, 2005, 2010, and 2015 as our data. Table 2 summarizes the data sources and periods.

\begin{tabular}{|c|c|c|c|c|c|}
\hline Period & I & II & III & IV & Source \\
\hline DALYs & 2000 & 2005 & 2010 & 2015 & $\begin{array}{l}\text { https://www.who.int/healthinfo/global_burden } \\
\text { _disease/estimates_country_2000_2015/en/ }\end{array}$ \\
\hline Articles \& Reviews & $2000-04$ & 2005-09 & $2010-14$ & $2015-19$ & Web of Science \\
\hline HDI & 2000 & 2005 & 2010 & 2015 & http://hdr.undp.org/en/data \\
\hline
\end{tabular}

Table 2. Data sources and periods 


\section{Research effort and disease burden - results and analysis}

The research framework in Figure 1 shows that three different analyses are required to answer our research questions: an overall analysis, followed by analyses by country, and another tracing international collaborations. These are presented in the following three subsections, with the results both divided into periods and the four human development levels.

\subsection{Overall analysis}

Note that all DALYs in this subsection are based on non-standardized total DALYs because differences between population sizes are not relevant to these global results.

\subsubsection{By IPD condition}

Figure 2 shows the disease burden and publication counts for the 11 IPD subcategories in four periods. With the steady improvement of health globally over the past 30 years (50), there has been a significant reduction in the global burden of IPDs in terms of DALYs. As for the effort side, the volume of IPD articles shows a clear increasing trend over the past 20 years as expected, with the general growth in the number of publications and journal coverage in the WoS (51).

Overall, the publications and burdens for 11 subcategories have maintained relatively stable shares within the IPD category, respectively. "Diarrhoeal diseases", "Parasitic and vector diseases", "HIV/AIDS" and "Childhood-cluster diseases" are the four conditions with relatively high burden through all the four periods. While, the distribution of publications for 11 subcategories is different from that of burdens, in which "Parasitic and vector diseases", "Hepatitis", "HIV/AIDS" and "Tuberculosis" count a high number of articles.

Two diseases that are prominent in both burden and effort sides are "Parasitic and vector diseases" and "HIV/AIDS". "Parasitic and vector diseases" accounted for the largest proportion of articles at nearly $29 \%$ on average. A number of neglected tropical diseases fall within this category, such as rabies, Chagas disease, leishmaniases, and schistosomiasis (52). These are responsible for a significant disease burden in impoverished children and adults worldwide (53). As for HIV/AIDs ${ }^{2}$, it continues to be a major global public health issue, with estimated 37.7 million people living with HIV at the end of 2020 (54). The scientific community globally paid relatively high attentions on HIV/AIDs, mightily due to its incurability, and the substantial and lasting negative social impact, especially in the hyperendemic countries of sub-Saharan Africa (55). Moreover, as a highly connected condition of HIV/AIDs, "Tuberculosis" also accounts for a non-negligible share of publications in IPD. Indeed, the weakened immune system caused by HIV will greatly increase the risk of tuberculosis in people with HIV (56).

"Hepatitis" is the second most widely concerned condition in IPD by academia. However, the burden caused by "Hepatitis" seems to be inapparent globally. "Hepatitis" itself is an inflammation of the liver with five main strains, referred to as hepatitis A, B, C, D and E. Yet, it can progress to or be complicated by other diseases. In particular, types $\mathrm{B}$ and $\mathrm{C}$ are the most common causes of liver cirrhosis, cancer and

\footnotetext{
${ }^{2}$ HIV stands for human immunodeficiency virus, which targets the immune system and weakens people's defense against many infections. The most advanced stage of HIV infection is acquired immunodeficiency syndrome (AIDS), which is defined by the development of certain cancers, infections or other severe long-term clinical manifestations.
} 
viral hepatitis-related deaths in hundreds of millions of people (57), which attracted extensive attention from academia. Further, China, as one of the high endemic geographic areas of hepatitis B virus (HBV) infection (58), had almost a third of the world's HBV carriers in the early $21^{\text {st }}$ century. With high potential needs on the prevention and treatment of this condition, relevant studies and trials were actively conducted by Chinese scientific community, which might be one of the causes of a high number of publications on hepatitis (59).

"Diarrheal disease" and "Childhood-cluster diseases" are two subcategories with remarkable burden but low level of research attention in IPD. "Diarrheal disease" is the second leading cause of death in children under five years old and is mostly caused by contaminated food and water (60). As a disease that is both preventable and treatable, it may be that alleviating the burden relies heavily on environmental improvement and access to treatment. "Childhood-cluster diseases" is a general term refers to several specific conditions, which are whooping cough, diphtheria, measles and tetanus. Among them, measles has the most remarkable burden with more than 140,000 measles deaths in 2018 globally (61). With the successful measles vaccine developed and improved in 1960s, it has become one of the best buys in public health to prevent measles. As a matter of fact, measles vaccination resulted in a $73 \%$ drop in measles deaths between 2000 and 2018 worldwide (62).

In addition, "Leprosy", an age-old disease with treatment scheme called multidrug therapy (MDT) (63), occupies the least proportion of publications in IPD. In fact, the burden of leprosy is also the lowest in IPD. "Encephalitis", and "STDs excluding HIV" also count a relatively low number of publications and burdens.
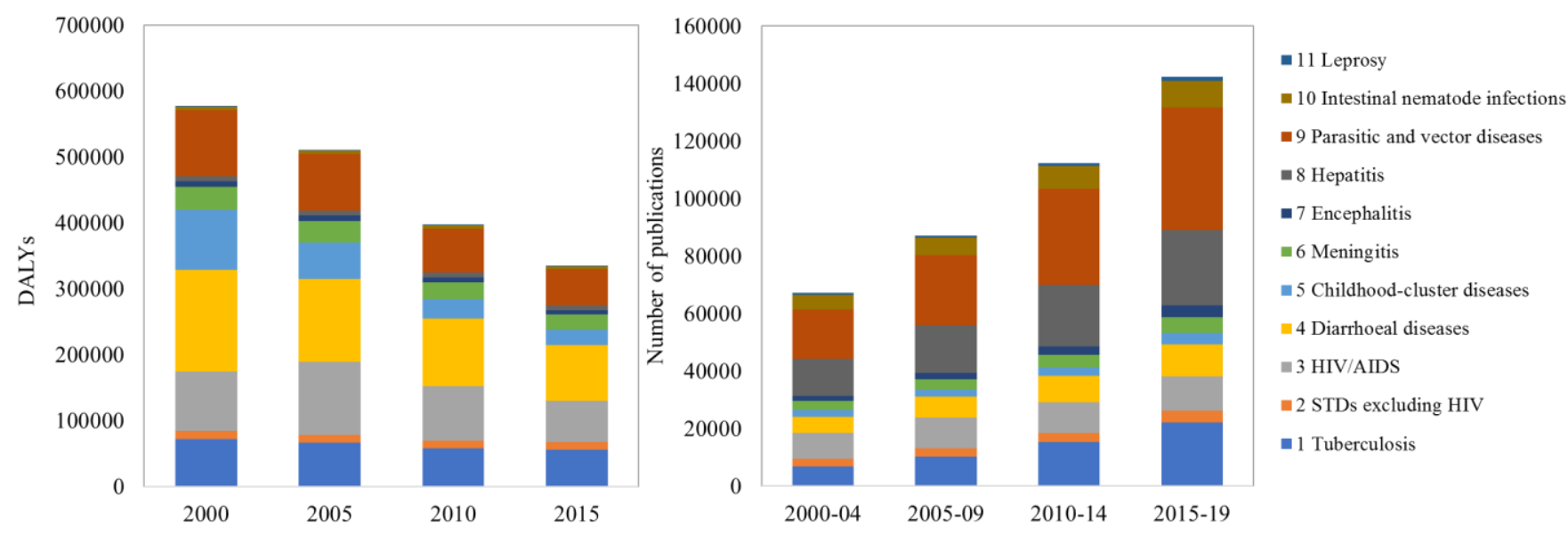

Figure 2. Disease burden and research effort by IPD condition in four periods

\subsubsection{IPDs in comparison to all diseases}

Here, the ratios of IPD research/burden as a fraction of all disease-specific research/burden are firstly calculated and compared to observe the "relative" degree of scientific response on IPD in comparison to all diseases globally. Furthermore, we also intend to investigate the correlation between IPDs' research effort and disease burden.

Figure 3 illustrates the distribution of publications and DALYs across the different time periods for IPD and its 11 subcategories compared to all diseases. In Figure 3(a), the bars and lines represent "original 
number" and "percentage of all causes" respectively. In general, although the number of publications on IPDs shows a steady increasing trend, the share of IPDs publication decreases over time (see the blue line with circles in Figure 3(a)). In terms of the burden side, a clear downward trend can be observed for both the absolute original DALYs and \% of DALYs.

(a) IPD in aggregate level

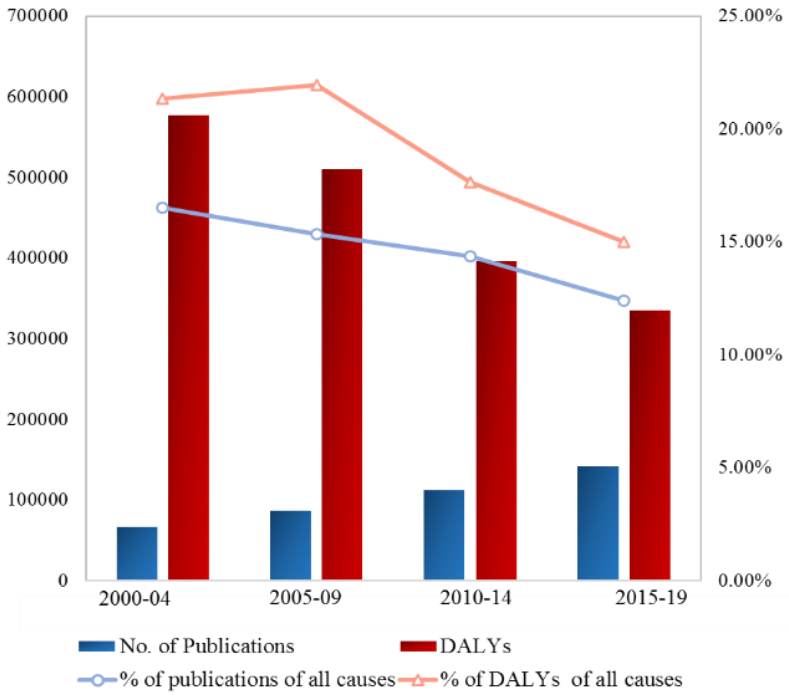

(b) 11 subcategories

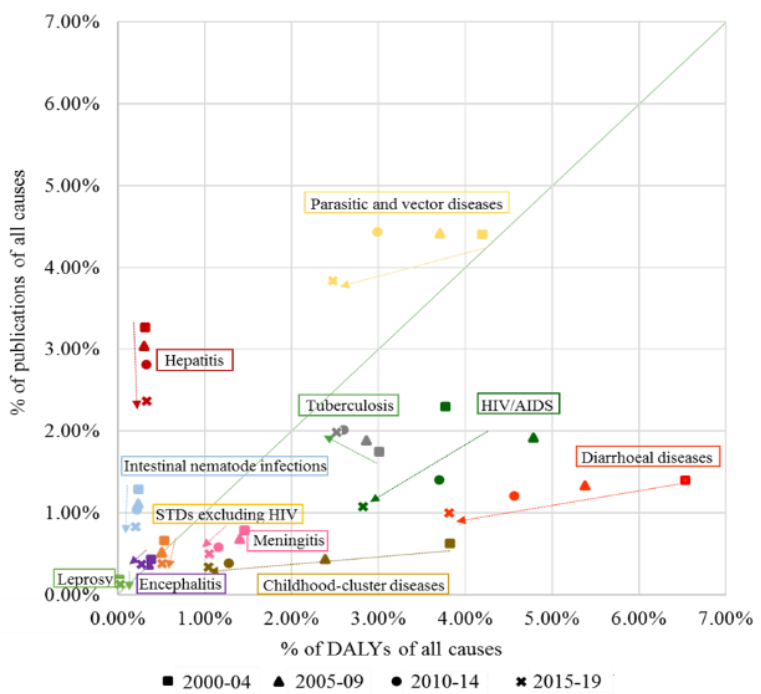

Figure 3. Research effort and burden of disease - IPDs vs. all diseases

Figure 3(b) illustrates a more fine-grained analysis of the share of disease burden (x-axis) versus the share of publications (y-axis) for the 11 subcategories. Here, the upper left corner of diagonal line indicates where the research effort exceeds the disease burden and vice versa. Corresponding to the general decreasing trend of IPD publications' share, the proportion of publications for all subcategories declined, except for "Tuberculosis", as shown in Figure 3(b). Together, the gap between research effort and disease burden does seem to be narrowing down, revealing also from the constantly approaching positions to the diagonal line of all 11 subcategories in Figure 3(b).

Figures 4 displays the logarithms of research effort versus the burden of disease for each of the 11 IPD subcategories in four periods. All graphs indicate a positive correlation, which means that, on average, the higher the disease burden, the greater the number of articles published. As revealed by the growing correlation coefficient for the four periods in general, research efforts on IPD tend to increases as time passes. Together, although the research efforts on IPD tend to decline in the form of share of all causes (Figure 3(a)), academia has positively responded to health need caused by IPD to some extent. 

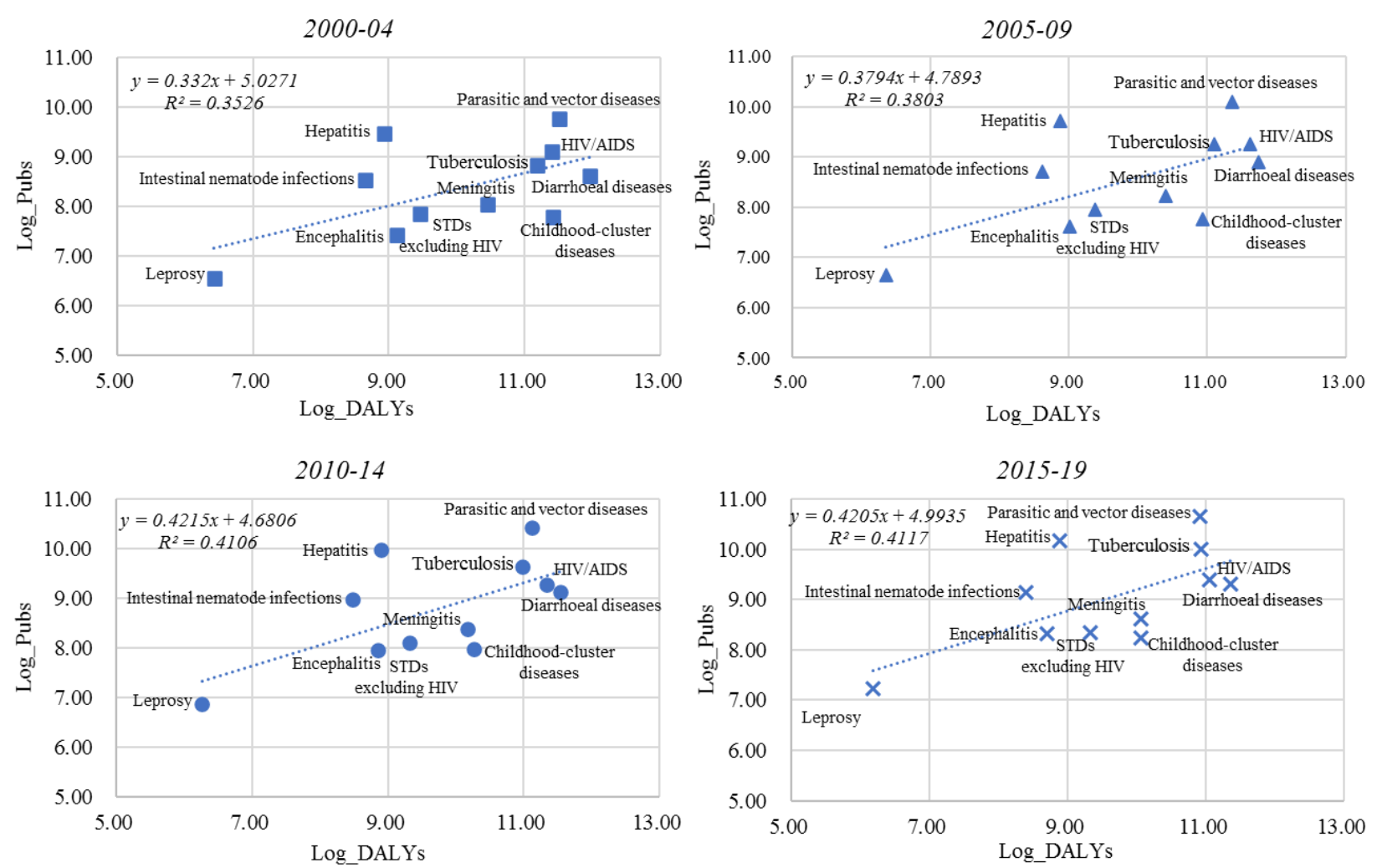

Figure 4. Disease burden (log) vs. research effort (log) by IPD condition

\subsection{By country}

Publications were assigned to a country according to the institutional address of each author with a full counting approach. In other words, an article was counted once for each country listed in the attributions. It is worth noting that the number of publications for different human development groups (countries/regions with various HDI levels) is counted directly on the aggregate level instead of summing the number of publications for each country/region within the specific HDI level, to avoid that publications collaborated by countries/regions within the same HDI level will be counted repeatedly.

\subsubsection{Overall}

As was to be expected, the countries with the highest disease burden are mainly concentrated in the less developed regions of Africa, with all of the top 40 countries with the greatest diseases burden (DALYs standardized 1000 per capita) situated on this continent. However, within these 40 countries, the particular conditions imposing the highest burden vary from country to country. Take the top 5 as an example (Sierra Leone, Central African Republic, Malawi, Zimbabwe, Lesotho), "HIV/AIDs" tops the list in Malawi, Zimbabwe, and Lesotho, while, in Sierra Leone and the Central African Republic, "HIV/AIDs" is nudged into later place by "Parasitic and vector diseases" and also "Diarrheal diseases".

The countries with the highest academic contributions are dispersed throughout the developed continents: America, Europe, Asia, and Oceania. Moreover, the top 3 countries with significant contributions are the traditional forces on academic publishing worldwide. Figure 5 shows the geographical distribution of research effort into the 11 different IPD conditions. Obviously, the US leads the world with an absolute advantage of almost 130,000 publications, followed by the UK, China, Brazil, and India. All these 
countries have relatively low burden on IPDs with ranking outside Top 100 list on DALYs per 1000 capita globally, except for India (ranked $55^{\text {th }}$ ).

What is noteworthy is that "Parasitic and vector diseases" is the most attended to category in most of the countries with high publication volumes, except for China. This type of IPD carries one of the highest burdens for Sierra Leone and the Central African Republic. While, research effort from China emphasizes diseases with a high potential local burden as mentioned above, such as "Hepatitis". "HIV/AIDs" has received substantial attention from scholars in the US and UK. "Tuberculosis" has also been a subject of interest for the top 5 publishing countries.

The tendency of developed countries to give attention to global health needs was verified in a prior study on the funding priorities of public funding agencies in the UK and China (17), Their results indicate that the UK funds a wider variety of research, extending to projects with impacts outside its borders and, among these, to some developing countries. Additionally, both public and non-public funding institutions in developed countries, such as the National Institutes of Health and the Bill \& Melinda Gates Foundation in the US, have established priority funds for research into the infectious diseases affecting the poorest regions and populations $(64,65)$.

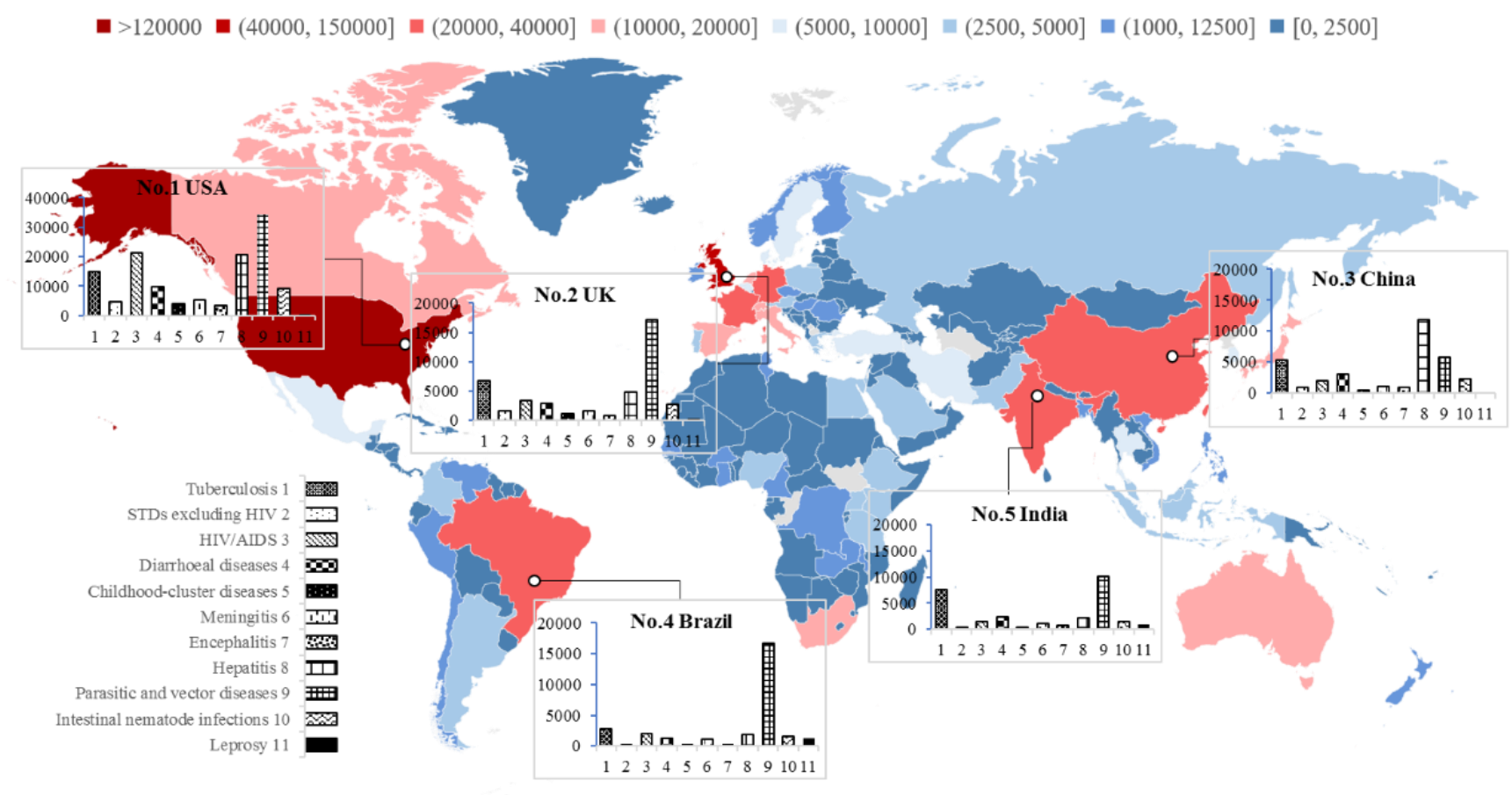

Figure 5. The geographical distribution of research effort - top five countries

\subsubsection{By human development level ('Absolute' global share)}

Note that the following is a dynamic analysis over 20 years, during which the development level of many countries is constantly changing. Hence, we chose to divide countries according to their dynamic HDI levels to reflect the research response pattern of regions with four HDI levels in the world against IPD. Further observation and interpretation of "fixed-level" countries and others with high impact on the results of global shares will also be provided in this section. Table 3 shows the number of countries falling into the low and medium development groups is decreasing over time. Accordingly, more and more 
countries are meeting the threshold for being classified as high and very high.

Table 3. Number of countries at different HDI levels in each of the four periods

\begin{tabular}{lcccc}
\hline HDI & $\mathbf{2 0 0 0 - 0 4}$ & $\mathbf{2 0 0 5 - 0 9}$ & $\mathbf{2 0 1 0 - 1 4}$ & $\mathbf{2 0 1 5 - 1 9}$ \\
\hline Low & 58 & 55 & 46 & 39 \\
Medium & 58 & 44 & 41 & 38 \\
High & 34 & 42 & 47 & 48 \\
Very high & 31 & 40 & 47 & 56 \\
\hline
\end{tabular}

Table 4 lists the disease burden and research effort for each of the four groups in each period, in which the proportion of the burden and publications of countries/regions in a given HDI level to the global burden and total number of publications are calculated respectively. Disease burden is shaded in green, with darker shades representing a greater burden of disease. Research effort is shaded in yellow, where, again, the darker the shade, the greater the research contribution.

Table 4. Global share of research effort and disease burden by human development level

\begin{tabular}{|c|c|c|c|c|c|c|c|c|c|c|c|c|c|}
\hline & & 金 & 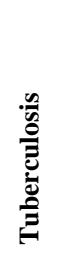 & 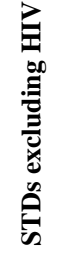 & 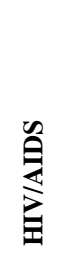 & 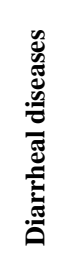 & 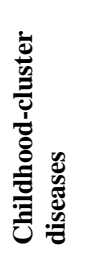 & 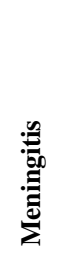 & 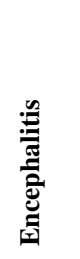 & 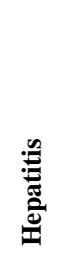 & 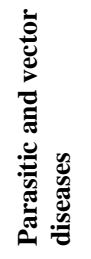 & 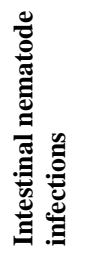 & $\begin{array}{l}\overrightarrow{0} \\
\stackrel{0}{0} \\
\text { 임 }\end{array}$ \\
\hline \multicolumn{14}{|l|}{ 2000-04 } \\
\hline \multirow{2}{*}{ Low } & DALYs & 85.50 & 73.46 & 76.45 & 77.47 & 88.15 & 93.61 & 87.64 & 80.36 & 73.36 & 94.44 & 40.96 & 79.49 \\
\hline & Pubs & 9.14 & 14.46 & 3.75 & 6.55 & 6.94 & 5.82 & 5.58 & 5.32 & 2.59 & 14.99 & 5.65 & 27.56 \\
\hline \multirow{2}{*}{ Medium } & DALYs & 12.98 & 23.05 & 19.63 & 8.43 & 11.12 & 6.29 & 10.47 & 15.87 & 21.25 & 5.34 & 55.68 & 19.96 \\
\hline & Pubs & 13.06 & 14.15 & 4.88 & 3.35 & 10.04 & 7.42 & 9.45 & 5.84 & 11.13 & 20.00 & 9.99 & 19.61 \\
\hline \multirow{2}{*}{ High } & DALYs & 1.06 & 2.99 & 2.02 & 1.68 & 0.60 & 0.09 & 1.22 & 2.66 & 1.83 & 0.19 & 2.88 & 0.37 \\
\hline & Pubs & 5.49 & 6.21 & 6.02 & 2.57 & 7.68 & 4.93 & 3.99 & 6.60 & 4.59 & 6.36 & 6.71 & 4.45 \\
\hline \multirow{2}{*}{ Very high } & DALYs & 0.46 & 0.50 & 1.90 & 1.34 & 0.12 & 0.02 & 0.67 & 1.12 & 3.56 & 0.03 & 0.48 & 0.17 \\
\hline & Pubs & 72.31 & 65.18 & 85.35 & 82.45 & 75.35 & 81.83 & 80.98 & 82.23 & 81.69 & 58.64 & 77.64 & 48.38 \\
\hline \multicolumn{14}{|l|}{ 2005-09 } \\
\hline \multirow{2}{*}{ Low } & DALYs & 84.01 & 75.01 & 77.40 & 73.69 & 89.28 & 91.90 & 89.76 & 80.73 & 76.87 & 93.97 & 43.75 & 77.50 \\
\hline & Pubs & 11.26 & 15.09 & 5.99 & 9.89 & 8.86 & 6.11 & 8.12 & 7.63 & 3.96 & 17.10 & 7.76 & 25.31 \\
\hline \multirow{2}{*}{ Medium } & DALYs & 13.20 & 19.16 & 16.33 & 21.99 & 9.13 & 7.87 & 7.63 & 14.42 & 17.20 & 5.34 & 49.96 & 14.46 \\
\hline & Pubs & 12.73 & 15.81 & 8.36 & 11.80 & 12.36 & 7.05 & 10.31 & 6.44 & 15.44 & 12.58 & 10.19 & 9.26 \\
\hline \multirow{2}{*}{ High } & DALYs & 2.26 & 5.29 & 3.79 & 3.33 & 1.34 & 0.20 & 1.97 & 3.47 & 2.89 & 0.66 & 5.91 & 7.87 \\
\hline & Pubs & 11.01 & 10.14 & 5.64 & 6.52 & 9.39 & 7.28 & 10.36 & 7.20 & 6.14 & 17.00 & 10.19 & 24.59 \\
\hline \multirow{2}{*}{ Very high } & DALYs & 0.53 & 0.54 & 2.47 & 1.00 & 0.25 & 0.02 & 0.65 & 1.38 & 3.04 & 0.03 & 0.39 & 0.18 \\
\hline & Pubs & 64.99 & 58.96 & 80.01 & 71.79 & 69.39 & 79.56 & 71.22 & 78.72 & 74.46 & 53.33 & 71.86 & 40.84 \\
\hline \multicolumn{14}{|l|}{ 2010-14 } \\
\hline \multirow{2}{*}{ Low } & DALYs & 57.19 & 34.54 & 58.87 & 57.66 & 57.85 & 57.57 & 69.06 & 23.80 & 23.52 & 80.03 & 22.83 & 19.53 \\
\hline & Pubs & 6.50 & 6.96 & 2.61 & 5.04 & 3.61 & 5.22 & 4.13 & 1.19 & 2.52 & 10.33 & 3.11 & 4.83 \\
\hline \multirow{2}{*}{ Medium } & DALYs & 38.36 & 59.40 & 31.19 & 34.19 & 39.84 & 41.54 & 26.83 & 68.19 & 69.78 & 18.79 & 64.51 & 69.85 \\
\hline & Pubs & 18.27 & 25.79 & 12.94 & 6.48 & 19.15 & 10.25 & 14.54 & 15.54 & 20.15 & 16.64 & 13.80 & 23.20 \\
\hline \multirow{2}{*}{ High } & DALYs & 3.69 & 5.37 & 6.42 & 6.99 & 1.81 & 0.80 & 3.16 & 6.00 & 3.99 & 1.06 & 12.02 & 10.21 \\
\hline & Pubs & 15.81 & 13.87 & 8.54 & 1.97 & 13.53 & 8.70 & 14.68 & 11.23 & 11.40 & 21.87 & 15.90 & 35.22 \\
\hline Very high & DALYs & 0.76 & 0.69 & 3.52 & 1.15 & 0.51 & 0.10 & 0.96 & 2.01 & 2.71 & 0.12 & 0.64 & 0.41 \\
\hline
\end{tabular}




\begin{tabular}{rlrrrrrrrrrrrr}
\hline & Pubs & 59.41 & 53.38 & 75.91 & 63.38 & 63.72 & 75.83 & 66.64 & 72.04 & 65.92 & 51.15 & 67.18 & 36.75 \\
\hline 2015-19 & & & & & & & & & & & & \\
\hline \multirow{2}{*}{ Low } & DALYs & 52.78 & 33.78 & 57.61 & 49.23 & 53.80 & 62.62 & 67.11 & 23.67 & 22.46 & 73.71 & 19.29 & 15.54 \\
& Pubs & 7.07 & 7.64 & 3.89 & 8.66 & 4.09 & 6.04 & 5.95 & 0.88 & 4.07 & 10.29 & 3.94 & 3.57 \\
& DALYs & 35.53 & 53.34 & 24.85 & 24.80 & 40.69 & 33.48 & 25.64 & 61.24 & 64.21 & 22.38 & 33.43 & 64.54 \\
\multirow{2}{*}{ Medium } & Pubs & 12.32 & 17.22 & 5.51 & 9.29 & 12.83 & 7.56 & 9.98 & 9.58 & 7.91 & 14.39 & 9.38 & 27.31 \\
& DALYs & 9.69 & 10.36 & 12.48 & 21.39 & 4.60 & 3.64 & 5.70 & 11.21 & 10.00 & 3.72 & 46.45 & 19.46 \\
\multirow{2}{*}{ High } & Pubs & 25.93 & 27.39 & 20.50 & 22.73 & 24.84 & 15.67 & 22.28 & 19.43 & 26.59 & 27.48 & 25.54 & 33.14 \\
& DALYs & 2.00 & 2.52 & 5.06 & 4.58 & 0.91 & 0.26 & 1.55 & 3.89 & 3.33 & 0.19 & 0.83 & 0.46 \\
\multirow{2}{*}{ Very high } & Pubs & 54.66 & 47.75 & 70.10 & 59.32 & 58.24 & 70.74 & 61.78 & 70.10 & 61.43 & 47.84 & 61.14 & 35.98 \\
\hline
\end{tabular}

Notes: DALYs are non-standardized. IPD is the aggregate total of the 11 subcategories.

Consistent with the design of the HDI, countries in the lowest group of human development carry the greatest disease burden. Likewise, the well-resourced infrastructures of countries in the very-high development group are responsible for contributing the most research to the literature. However, despite a steady increase in the number of publications produced by the very high group across the four periods, the proportion of research effort they are contributing is declining. In other words, the number of articles on IPD produced by the other groups is also increasing and at a greater rate. In fact, the proportion of publications generated by those traditional highly developed countries (i.e., 31 countries in the very-high group in all four time periods) declined in a more significant manner from $72.31 \%$ to $48.29 \%$ (see Table 5). In contrast, the contribution of countries "always" in the low HDI group, i.e., 39 countries in the low group in all four time periods, is clearly and substantially increasing (from $3.63 \%$ to $7.07 \%$ ), which speaks to a persistent effort by scholars in the areas affected to do something to ease their high local burden.

Special attention is given to countries in the "always" low HDI level, who are also the ones with high burden on IPD, to further observe their scientific response pattern to local burdens. In addition to the constant increasing number of publications mentioned above, the distribution of research efforts from "always" low group on the 11 specific conditions also corresponds with the distribution of burdens in those regions to some extent, as shown in Table 5. In particular, "Parasitic and vector diseases" caused the highest burden among all the 11 conditions in terms of both original values and global shares for the "always" low group. Correspondingly, publications on this disease accounted for the highest proportion for those undeveloped regions. Moreover, although "Parasitic and vector diseases" is already the most concerned type among 11 diseases by scholars around the world (Figure 2), the proportion contributed by the "always" low group in terms of the whole world is still increasing from $7.41 \%$ to $10.29 \%$, as Table 5 shows. In spite of the general growing share of publications in IPD, "Leprosy" is the only disease with a sustained decline in the proportion of publications in the last two decades for the "always" low group, which is also the one caused least burden in those regions. In sum, those undeveloped areas have contributed concentrated and specialized efforts to ease their local burdens. In the context of the growing literature on IPD globally, the proportion of publications contributed by those underdeveloped regions is increasing remarkably. As for the burden side, although the global share of DALYs for the "always" low group fluctuate slightly around 52\% (Table 5), an obvious downward trend of DALYs' original value of those regions can be observed in the last two decades. 
Table 5. Global share of research effort and disease burden for "always" low and very-high group

\begin{tabular}{|c|c|c|c|c|c|c|c|c|c|c|c|c|c|}
\hline & & $\hat{\mathrm{E}}$ & 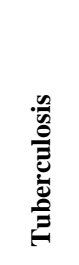 & 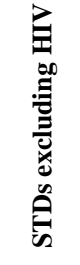 & 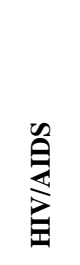 & 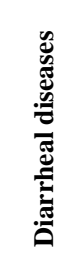 & 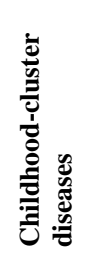 & 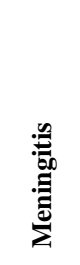 & 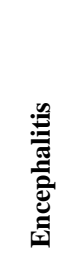 & 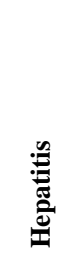 & 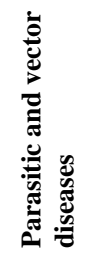 & 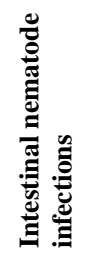 & \\
\hline \multicolumn{14}{|l|}{ 2000-04 } \\
\hline \multirow{2}{*}{ Low } & DALYs & 51.95 & 26.48 & 41.17 & 48.25 & 46.33 & 64.72 & 58.14 & 19.09 & 19.27 & 78.04 & 13.36 & 12.76 \\
\hline & Pubs & 3.63 & 4.92 & 1.76 & 0.90 & 1.37 & 2.82 & 2.15 & 0.29 & 0.57 & 7.41 & 1.44 & 6.62 \\
\hline \multirow{2}{*}{ Very high } & DALYs & 0.46 & 0.50 & 1.90 & 1.34 & 0.12 & 0.02 & 0.67 & 1.12 & 3.56 & 0.03 & 0.48 & 0.17 \\
\hline & Pubs & 72.31 & 65.18 & 85.35 & 82.45 & 75.35 & 81.83 & 80.98 & 82.23 & 81.69 & 58.64 & 77.64 & 48.38 \\
\hline \multicolumn{14}{|l|}{ 2005-09 } \\
\hline \multirow{2}{*}{ Low } & DALYs & 51.13 & 28.44 & 46.40 & 45.83 & 47.97 & 61.26 & 63.71 & 21.12 & 20.87 & 77.34 & 15.55 & 13.60 \\
\hline & Pubs & 4.35 & 4.29 & 2.85 & 5.39 & 1.82 & 3.52 & 2.71 & 0.48 & 1.36 & 8.24 & 2.03 & 4.12 \\
\hline \multirow{2}{*}{ Very high } & DALYs & 0.47 & 0.43 & 2.19 & 0.89 & 0.24 & 0.02 & 0.57 & 1.22 & 2.92 & 0.03 & 0.38 & 0.18 \\
\hline & Pubs & 61.74 & 58.07 & 78.45 & 71.38 & 67.87 & 78.66 & 70.15 & 78.20 & 73.52 & 52.81 & 70.30 & 40.53 \\
\hline \multicolumn{14}{|l|}{ 2010-14 } \\
\hline \multirow{2}{*}{ Low } & DALYs & 51.01 & 30.17 & 52.63 & 47.79 & 51.56 & 52.86 & 63.39 & 20.29 & 21.23 & 75.43 & 17.70 & 14.94 \\
\hline & Pubs & 5.64 & 6.16 & 2.45 & 2.66 & 3.36 & 5.09 & 3.83 & 0.46 & 2.42 & 9.35 & 2.62 & 3.39 \\
\hline \multirow{2}{*}{ Very high } & DALYs & 0.53 & 0.38 & 2.50 & 0.83 & 0.42 & 0.03 & 0.61 & 1.42 & 2.34 & 0.03 & 0.38 & 0.19 \\
\hline & Pubs & 55.02 & 51.22 & 73.10 & 63.68 & 60.76 & 73.46 & 64.24 & 68.55 & 63.61 & 49.00 & 63.48 & 35.99 \\
\hline \multicolumn{14}{|l|}{ 2015-19 } \\
\hline \multirow{2}{*}{ Low } & DALYs & 52.78 & 33.78 & 57.61 & 49.23 & 53.80 & 62.62 & 67.11 & 23.67 & 22.46 & 73.71 & 19.29 & 15.54 \\
\hline & Pubs & 7.07 & 7.64 & 3.89 & 8.66 & 4.09 & 6.04 & 5.95 & 0.88 & 4.07 & 10.29 & 3.94 & 3.57 \\
\hline \multirow{2}{*}{ Very high } & DALYs & 0.59 & 0.36 & 2.48 & 0.89 & 0.60 & 0.03 & 0.59 & 1.55 & 2.23 & 0.04 & 0.36 & 0.21 \\
\hline & Pubs & 48.29 & 43.55 & 65.21 & 56.55 & 51.80 & 65.69 & 57.56 & 63.15 & 55.48 & 44.32 & 55.76 & 30.48 \\
\hline
\end{tabular}

Notes: DALYs are non-standardized. IPD is the aggregate total of the 11 subcategories.

A closer look at specific countries behind the HDI groups might provide more in-depth insight on how least developed countries defend themselves against the threats of infectious diseases. Indeed, 6 of the 10 African countries with highest number of publications are classified constantly in the low HDI group. Specifically, 4 of the 6 countries are still on the latest "UN (United Nations) list of least developed countries" (66). Among them, Uganda is the one with the largest publication outputs, who also bears high burden on IPD (ranked $8^{\text {th }}$ and $9^{\text {th }}$ of DALYs in original value and per 1000 capita respectively). With the continuous growing proportion of publications on IPD, Uganda's global ranks of DALYs in both original value and per 1000 capita forms declined significantly from 2000 to 2019, illustrating a greater reduction in IPD burden compared to the overall global decline. In terms of the specific diseases, "Parasitic and vector diseases" and "HIV/AIDs" are the two major causes of such high burdens of Uganda. Accordingly, among IPD publications produced by Uganda, almost 70\% of publications targeted on these two diseases. As a matter of fact, Uganda has been widely regarded a one of the world's earliest and most compelling national success stories in combating the spread of $\operatorname{HIV}(67,68)$, who has experienced the sharpest decrease in HIV/AIDS-related death rate in the world between 1990 and 2017 (69). In addition to the massive efforts on scientific research, Uganda has established a comprehensive HIV/AIDS program with the high-level political support and multi-sectoral response (70). Indeed, both the scientific research and policy implementation is inseparable from international assistance. Early in 1986, representative of Uganda informed about the domestic HIV/AIDS pandemic on the World Health Assembly (WHA) and 
called for the support and help from international community (71). Its high dependency on international collaboration can be revealed by the high share of international collaborative IPD publications (90.26\%), especially with highly-developed countries (i.e., 50.41\% of publications are collaborated with the USA). As for the "Parasitic and vector diseases", corresponding to the steady rise in the number and global share of publications targeting this disease, Uganda's burden decreased remarkably in terms of both global share (from $5.18 \%$ to $2.48 \%$ ) and ranking (from $4^{\text {th }}$ to $11^{\text {th }}$ ).

In addition to the conspicuous patterns of the very-high and low group, the shares of publication and burdens for high and medium ones seem like to fluctuate more erratically. Generally speaking, in most periods except for the most recent, the proportion of publications produced by countries in the high group was even lower than those in the medium one. Obviously, medium-group countries carry a greater burden of disease to IPD, and so have a stronger incentive to address the subject. Another possible explanation goes with further digging into the data - China moved from the "medium" group to the "high" group in the last period. With the full measure of China's output counted in the medium bracket up until then, it is no wonder that high-group countries could not compete. Indeed, the medium and high levels are the two with the most frequent "mobility" in countries. The change in HDI levels of countries with high absolute value of disease burden (and large population) and publication volume will greatly affect the results of these two levels.

In particular, India, a country with large population, has the highest original DALYs across the whole study periods on the aggerate IPD level. Hence, the upgrade of India from low to medium level in third period inevitably leads to the increasing share of burdens for medium groups, especially for several disease with highest burden in India ("Tuberculosis", "Encephalitis", "Hepatitis" and "Leprosy"). Moreover, China also has the relative high value of original DALY in "Encephalitis" and "Hepatitis" (both ranked among the top 5 globally). Indeed, although China's burdens on those two diseases is lower than India's, the large volume of publications generated by Chinese scholars, together with China's movement from medium to high group in the last period, resulted in the growth share of publications of high group. As for "Intestinal nematode infections", China ranks top 1 in original DALYs from 2000 to 2019, which explained the high global shares of burden in medium group during 2000-04 and its substantial increase in high group in 2015-19. Correspondingly, the intensive effort from China on "Intestinal nematode infections", especially in the latest period, contributes to a remarkable rise of publications' share for high group in 2015-19. Different from the above-mentioned diseases, the increasing share of both burden and publications for "HIV/AIDS" of high group in 2015-19 mainly due to the upgrade of South Africa from medium to high group in the same period. On average, South Africa ranked the $1^{\text {st }}$ and $3^{\text {rd }}$ in burden and publications respectively, which also partly reveals its high research attention on local health needs.

In spite of the highest share of burden and publications in low and very-high group respectively, the tendency of giving priority to local health needs can be observed not only from the steady increase of "always" low group's share of publications, but also from the intensive research efforts on disease with high local burden from several major countries with "mobility" in HDI levels. This conclusion, however, does not consider the effort a country extends toward IPD as a proportion of all its disease-related research. Thus, one may reach a different conclusion when examining how a nation balances its research efforts between local and global needs. Such analysis needs to be based on a country-specific perspective, 
combined with the information of burden and research effort for all categories of diseases instead of only IPD. This is somewhat beyond the scope of this paper, as we are focused on the research effort on IPD of countries toward not within each country and their specific research priorities. That said, this reasoning raises the point that our results cannot be taken at face value. Rather, they must be interpreted more carefully from a multi-dimensional and dialectical perspective. Indicators such as the percentage of country's publications devoted to IPDs relative of that of the world cannot accurately reflect the true machinations of specific countries' research concerns. A more fine-grained analysis is needed to address this problem.

\subsubsection{By human development level ('Relative' specialization index)}

Following the global share of disease burden and research effort calculated based on original absolute numbers, this section adjusts the two factors to reflect "relative" level of burden and efforts in terms of the world average without the impact of population size.

Figures 6 and 7 show the disease burden and research effort, as calculated by the normalized specialization index (NSI) for the different human development groups. The results for disease burden (Figure 6) are similar to the previous analysis, with countries in the low group carrying a greater burden than the world average. Moreover, over the full period, this tendency has only strengthened, with the value of NSI_DALY rising for both low and medium groups. So, although the burden of IPDs has decreased overall, the problem for many low-to-medium HDI level countries is still serious. One possible explanation for an increased burden on disease in the medium group is that more than 10 countries who were previously in the low group had their status upgraded in the later periods. It would not be surprising to find that IPDs have not been alleviated even if other indicators of human development have risen. Several diseases in Figure 6 show a particular tendency to shift from low to medium and even to the high groups, which are basically the similar ones as discussed above - "Encephalitis (7)"3, "Hepatitis (8)", "Intestinal nematode infections (10)" and "Leprosy (11)".

Figure 7 shows the research effort for different groups, where we find the patterns of the previous analysis amplified - low-level countries are clearly exerting greatest effort to research IPDs, with the only exception of "Encephalitis (7)". As mentioned, the overall number of publications in "Encephalitis (7)" is the second lowest among all 11 conditions, in which India contributed the majority for low group in the first two periods. Nevertheless, a strong conclusion drawn from this result is that low-level countries are striving hard to ease their own burdens. Another notable point is that the value of NSI_Pub for the high group was higher than 0 in most cases. This means that those countries appear to be expending more effort to explore IPDs than the world average.

\footnotetext{
${ }^{3}$ The number in parentheses after the name of a disease corresponds to the number on the horizontal axis of Figure 6 or Figure 7.
} 

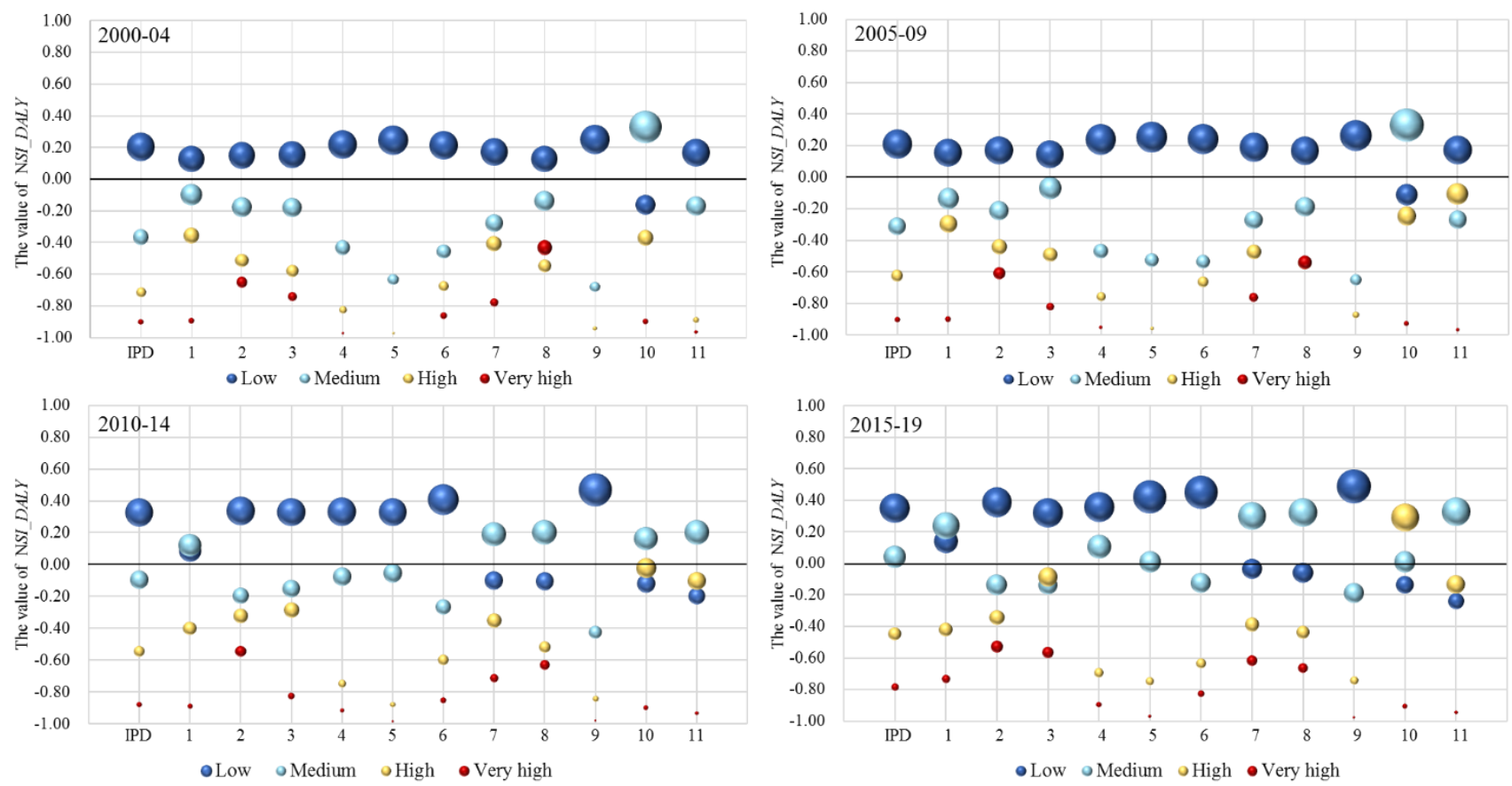

Figure 6. NSI_DALY by human development group

Notes: IPD is the aggregate total of the 11 subcategories. The category names for Codes 1 to 11 are given in Table 1 . The size of the bubbles represents the disease burden.

What seems contrary to the above analysis is that, the very-high group is making the least research contribution (see red bubbles in Figure 7). In examining the data behind these averages, almost every country made less of an effort than the global average. However, even at the least effort, the number of articles published absolutely blitzes any other group. For all diseases, very-high-level countries generate $75.7 \%$ of the total articles. For IPD-related articles, that number is $60.6 \%$. In other words, in absolute terms, the world average is highly influenced by the very high group. Hence, NSI_Pub of the very high group will naturally fall below 0 when countries in low group concentrated more on IPD instead of other types of diseases within their research capability.

We now turn to the balance of research effort between global and local needs. Since low-level countries are exerting such a strong effort to research IPDs, we can also surmise that they are prioritizing local issues. In terms of the other three groups, it is difficult to argue for any firm conclusions without a thorough and detailed exploration of the other disease burdens they may be facing. However, as the figures for research effort across all diseases show (see Appendices A2), the very high group tends to focus more on non-communicable diseases, like neurological conditions and cardiovascular disease. These are diseases of high burden in developed countries (50).

The tendency on focusing more on local health needs has been verified for both developed and undeveloped regions in the above analysis. Again, however, we must highlight that an integrated exploration of burden and research efforts on all types of disease for specific countries is needed to reflect detailed information on the balance between global and local needs. Yet, what emerges clearly from this analysis is the complexity of setting research priorities and establishing a research agenda by referring to quantitative indices. Taking our results as an example, if one relies only on the results produced by the absolute 
calculations, then practical issues such as the actual research capacity of each country might be overlooked. Under such circumstances, analyzing the quantitative outcomes from multi-dimensional aspects and integrating them with qualitative analysis may help to develop more rational and effective policies.
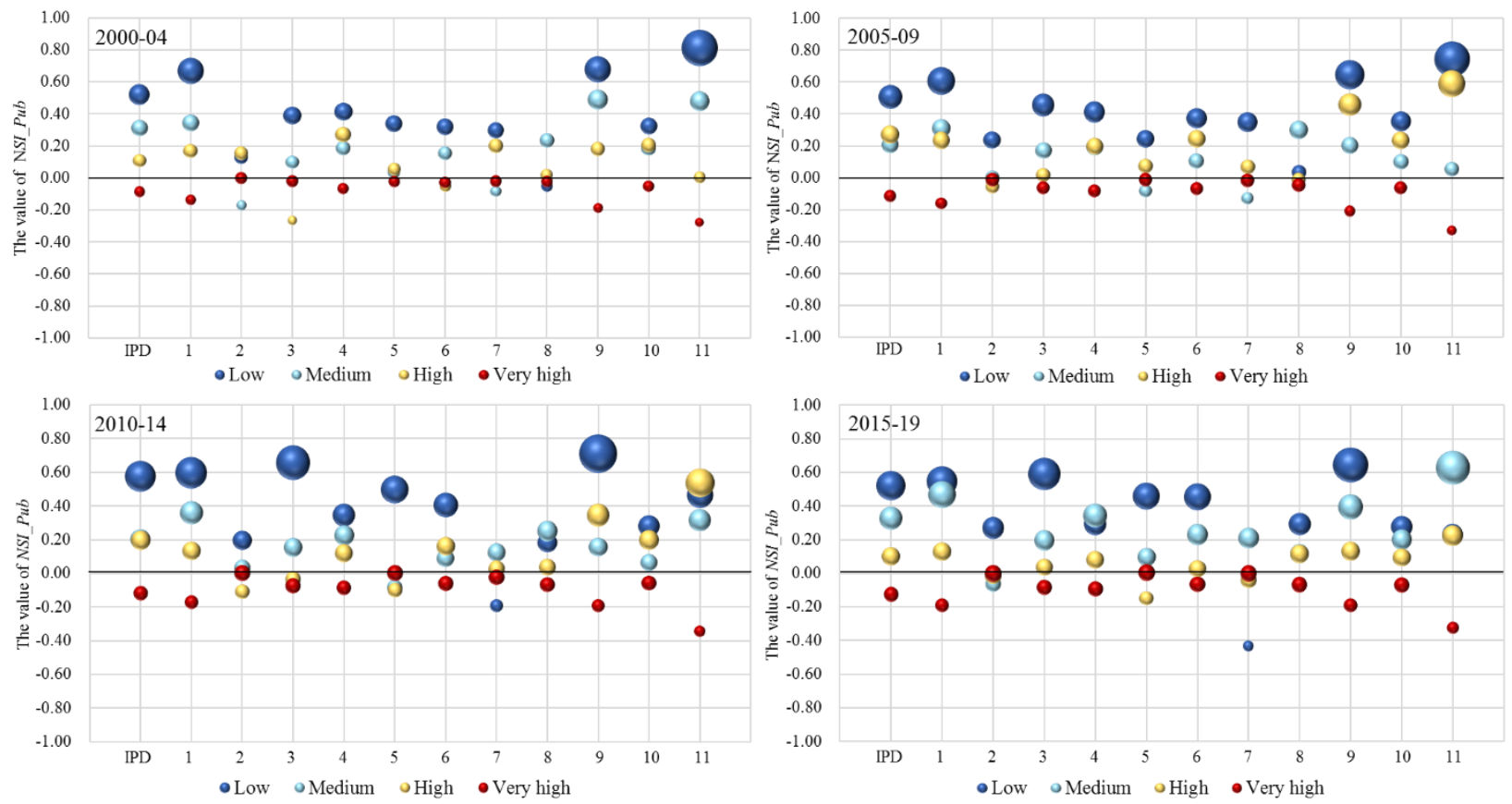

Figure 7. NSI_Pub by human development group

Notes: IPD is the aggregate total of the 11 subcategories. The category names for Codes 1 to 11 are given in Table 1 . The size of the bubbles represents the research effort.

\subsection{International collaborations}

This section provides an analysis of the collaboration patterns in two respects: overall and with respect to the countries with a high disease burden. Collaborations are measured both in terms of the number of joint publications and the strength of collaboration. Salton's measure (72) is used to calculate the strength of collaborations, which is defined as the number of joint publications divided by the square root of the product of the total publications of the two countries (i.e., the geometric mean).

\subsubsection{Overall}

Among all the countries that have involved in the scientific publishing on IPDs, 65 have generated more than 1000 publications, and these we selected as the major subjects of study in this section. Figure 8 shows a collaboration network of these 65 countries, visualized through VOSViewer (73). 


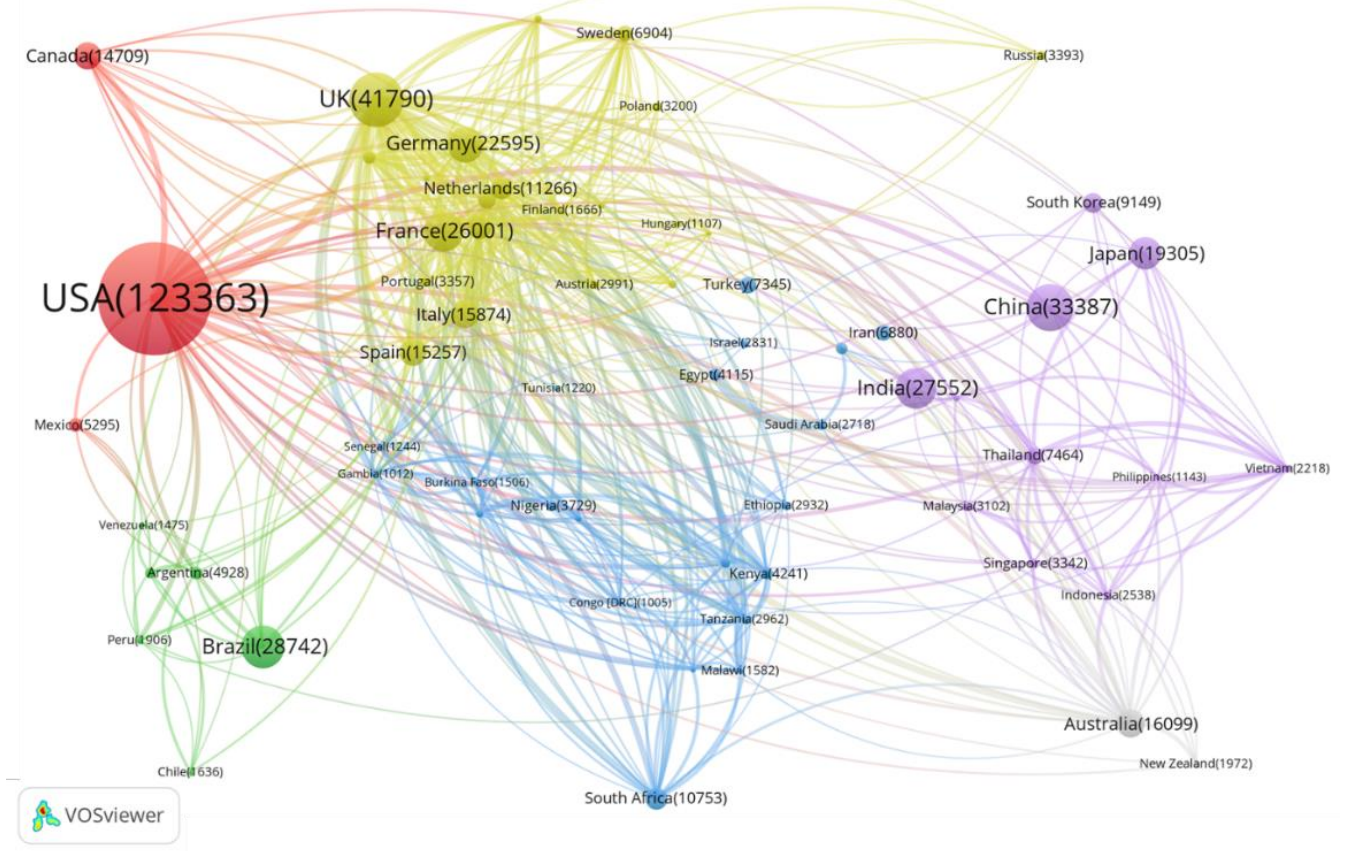

Figure 8. International collaboration patterns

Notes: The size of the node indicates the number of publications produced by the country, which corresponds to the number presented on the node. The thickness of the links reflects the strength of the collaboration between the two countries. The different colors relate to the continent a country is situated on.

Collaborations occur on all continents ${ }^{4}$, especially in Europe. With the largest publication total, the US has a significant number of collaborations with countries from all the other continents. Scholars in the UK work closely with researchers both in neighboring countries and in Africa to tackle the public health needs raised by IPD. Although ranked in third for research effort, China generated many fewer links than the US and UK, and Chinese scholars seem more inclined to collaborate with scholars from other Asian countries. What is promising is that in Africa, 21 countries have published more than 1,000 papers, of which South Africa leads the list with more than 10,000 papers. Further, intensive collaborative studies have also been conducted within the African content and with the West.

In order to further explore the variation trends of collaboration patterns worldwide, the number of collaborative publications and collaboration strength are calculated for six "collaboration pairs" generated by four HDI groups in different time periods, as shown in Figure 9. Here, each colored annular sector represents a "collaboration pair" and the distance between outer arc length and inner arc length of each sector depict the number of collaborative publications or collaboration strength for that pair. The colors and corresponding pairs are given on the left of Figure 9. From the comparative aspect, as expected, the most collaborative countries are those in the very high human development group. This group has three country pairs. However, collaboration strength is highest between the very high and the low group, although the other two pairs have the larger number of collaborative publications. It is worth noting that the collaboration between very high and high countries significantly intensified post-2015 when China shifted groups. High-low pairs were the least cooperative.

\footnotetext{
${ }^{4}$ Antarctica excluded.
} 
(a) The number of collaborative publications

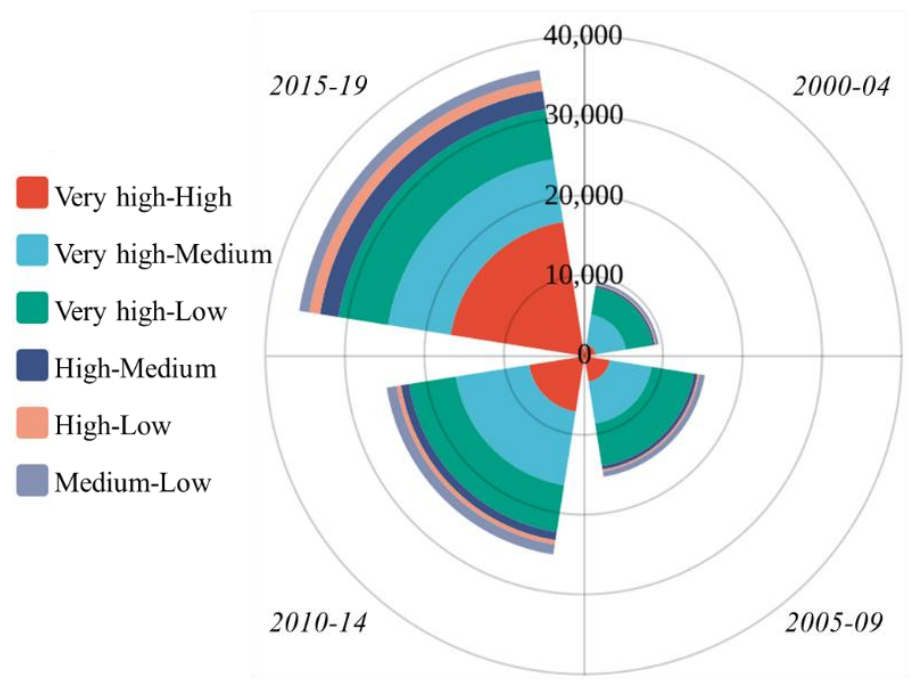

(b) The strength of collaboration

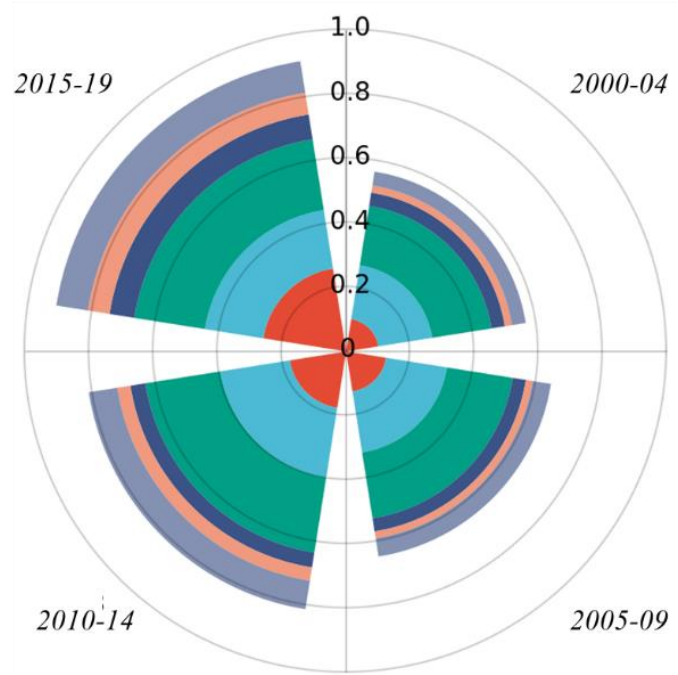

Figure 9. Collaboration patterns between HDI groups by time period

From the aspect of time evolution, both the number of collaborative publications and the strength of the collaborations increase over the period, especially for the collaboration pair of "very-high and high group" (the red sector). With the general increase in the number of IPD publications, it is quite natural to observe the growing volume of collaborative publications. As for the closer collaborative relations observed among different collaboration pairs, further observations are made from the perspective of number of HDI groups involved in one publication. The ratio of publications generated within the same HDI group ${ }^{5}$ experienced a steady decline from $86.46 \%$ to $78.27 \%$ during the four time periods, indicating the increasing proportion of "cross-HDI level" collaborative publications. Specifically, the ratios of "bilateral" collaborative publications generated between two HDI groups for the six pairs also show downward trends from 2000-04 to 2015-19 in general. Obviously, the more frequent "multilateral" collaboration among three or four HDI groups also contributes to a higher value of collaboration strength in the later period.

\subsubsection{With respect to disease burden}

As mentioned, high-burden countries are concentrated in Africa. However, among the 21 countries on the African continent with more than 1000 publications, only Malawi and Nigeria are in the top 10 list of countries with the greatest burden of IPD, ranked $3^{\text {rd }}$ and $6^{\text {th }}$, respectively. To gain further insight on how countries with highest burden on IPD conduct relevant research, the five countries with the highest burden (countries with * in Figure 10) are regarded as the objects to generate their global collaborative network with VOSViewer, as shown in Figure 10.

It is unsurprising to see that European countries occupy a significant position in collaborating with highburden countries, especially the UK, which has demonstrated a strong partnership with Malawi. The US is also a key partner to these countries as well as with Zimbabwe. Another quite remarkable point is the

\footnotetext{
${ }^{5}$ A publication generated within the same HDI group could refer to the output of a single country within this group or a collaboration of multiple countries within this group.
} 
cohesive collaboration between African countries. South Africa, as a key academic contributor in Africa, plays a vital role in the collaborative networks of high-burden countries. Swaziland and Lesotho have the strongest collaboration. This is perhaps to be expected since the two countries are highly similar in geographical location, language, and culture. Compared to other continents, collaborations between Asian countries and Africa are relatively weak. Notably, China's collaborations with the high-burden countries do not even match Mongolia, Thailand or several other countries in the region.

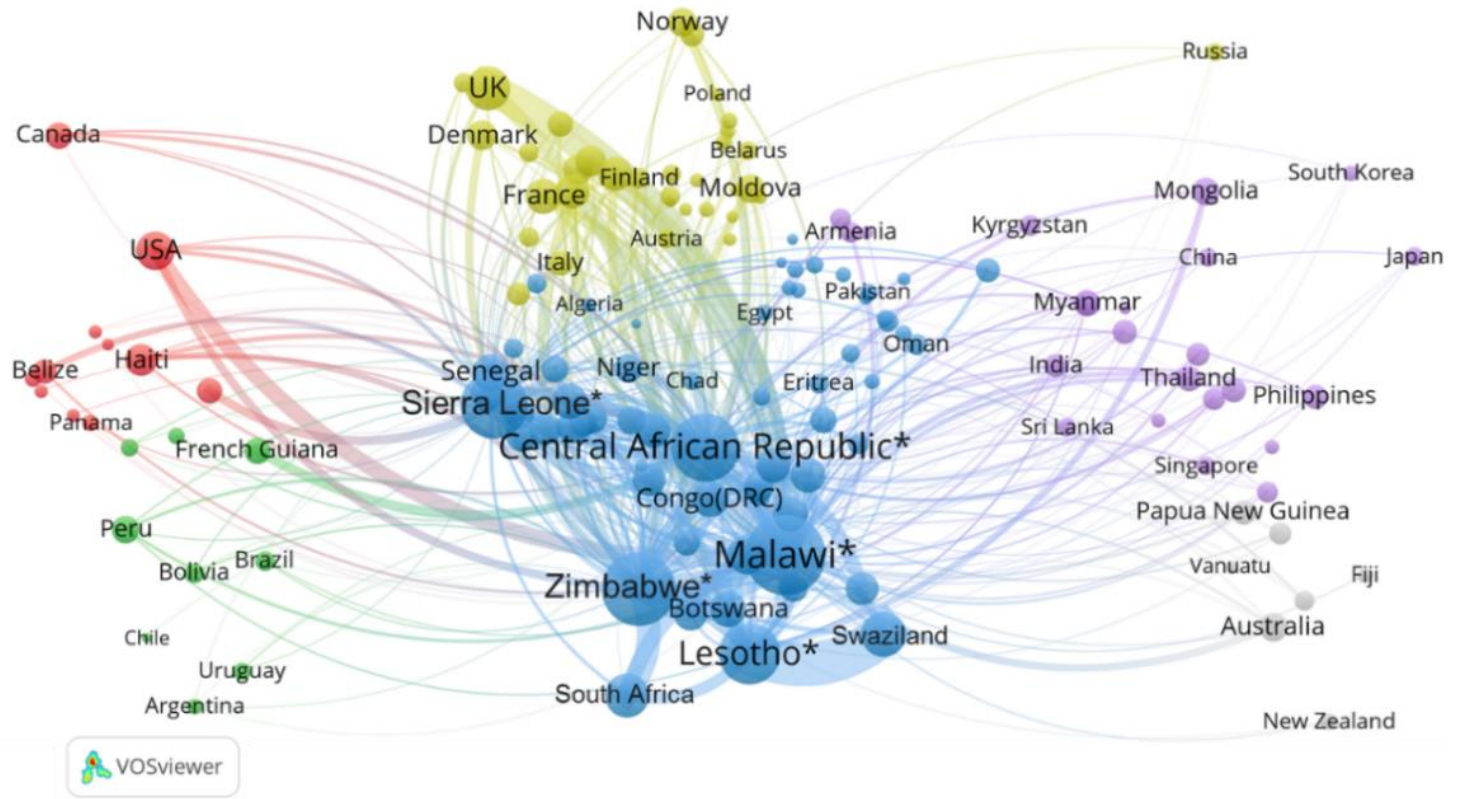

Figure 10. Collaboration network of the five countries with the greatest disease burden

Notes: The size of the node indicates the number of the other countries a nation collaborates with. The thickness of the links reflects the strength of the collaboration between the two countries. The different colors relate to the continent a country is situated on.

From the aspect of aggregate level of HDI groups, the strongest collaborators over the past two decades with the five greatest-burden countries have been those in the very high group, followed by those in the low group who also suffer from great IPD burden. Together with the results from Section 3.2, this analysis further reveals that the most research is produced by African countries focused on their local burdens. However, enriching this insight is the findings that some of this effort involves relatively strong collaborations with highly-developed regions. This implication confirms Confraria and Wang's (30) conclusion that there is no clear trade-off for African countries between participating in global research networks and producing medical research that is aligned with local health needs.

\section{Conclusion}

\subsection{Summary}

IPDs are one of the leading causes of death and disability and are responsible for a significant burden on public health and social progress. As an essential means by which human beings can fight against various diseases, how academics react to the severe health needs caused by IPDs is a vital question to be explored. Thus, this international review of what, where, and how research has been done and how it matches with societal needs is an insightful source of knowledge for ensuring an efficient global research effort. 
In this paper, we investigated the response patterns of academic research to IPDs from the dynamic relationship between disease burden and research effort on IPD, the scientific efforts contributed by countries with different development levels, and the variation trends in international collaborations. Our finding reveals a substantially diminishing gap and a positive correlation between the level of research efforts and burden globally. In terms of the regional distribution, high IPD disease burden is mainly concentrated in the less developed regions in Africa, while related academic research is mainly from developed continents. Although specialization index indicated that those highly developed countries contribute less than the world average level, in terms of absolute number of publications, they obliterate the competition, accounting for nearly two-thirds of all outputs. More importantly, there is a clear increase of research efforts from regions with the heaviest disease burden, despite their limited research capacity. In fact, apart from the constant increasing number of publications produced by the least developed regions, the distribution of their research efforts on the 11 specific conditions also relatively corresponds with the distribution of burdens in those regions, in which the "Parasitic and vector diseases" is the one with the highest burden and receives the most attention. In particular, Uganda stands out as a compelling example of paying diligent attention to its local health needs and achieving remarkable effects on alleviating their burdens.

We also noted that there is no uniform standard or requirement for academic contribution for each country across the different development levels. The uneven distribution of research effort by the different nations once again raises the issue of whether or not research effort should be balanced between global and local needs or whether a local focus is optimal. Although this question is not the major focus of our research, our analysis reveals a tendency for all countries of the world, no matter their development level, to concentrate on their own issues. This is a finding that is consistent with previous studies $(26,38)$ but one that, no doubt, will stoke further lively debate.

As for academic collaboration, our analysis shows that both the number of collaborative publications and collaboration strength has increased remarkably in the past two decades across the board. Collaborations are most intense between the very high and low groups. In particular, the US and UK play a prominent role in collaborating with high-burden countries. Moreover, the cohesive collaboration within African countries can also be observed from this study. Among all the collaboration pairs, the high-low collaborations generate the least publications and are weakest. Additionally, China, a high (medium)level country, has a relatively weak collaboration with high-burden countries despite its place as the third highest research contributor.

\subsection{Limitations and future research}

This study contains several limitations that need to be addressed. First, the only measure of research effort was publication counts from WoS. Drawing only from WoS might mean the corpus did not include some relevant publications indexed in other scholarly databases. Moreover, while publication counts are an objective measure, they don't represent all types of research efforts. The same is true for the burden of disease. A wide range of indicators beyond DALYs have been developed to monitor and manage health initiatives $(74,75)$. Using these indicators may produce different results. Hence, these empirical results need to be interpreted and applied to policymaking with caution due to their different computing logics, limitations, and applicable scenarios. 
Second, it is a controversial discussion as to whether research effort and academic resources should be allocated relative to the health needs or more directly to the burden of disease (76). A range of factors influence what types of research gets priority. Additionally, alleviating the burden of disease and addressing health needs does not just involve an endless research effort, it also relies on the appropriateness of practices, access to the prevention and treatment facilities, medical quality controls and the financing of healthcare system. Hence, we acknowledge that measuring the association between research effort and disease burden is not straightforward; multiple societal factors affect both sides. In practice, it might be impossible to quantify the optimal relationship between a disease and its corresponding R\&D efforts for any disease or group of diseases. Nevertheless, gaining a thorough and systematic view of the dynamic relationship between research efforts and health needs is the first step to understanding the complex, multi-dimensional, and integrated interaction mechanisms between science and society. Moreover, it is undeniable that this is the most transparent and fair way to quantitatively explore the dynamic relationship between health needs and research effort. Such insights are useful when setting priorities for R\&D investment (48) and for providing explicit empirical evidence on these issues to policymakers and the public (43).

For future research, multi-dimensional indices should be expanded to provide a more comprehensive understanding of the relationship between research efforts and societal demands in medical fields. Diverse societal factors relevant to health needs could be included to investigate the more in-depth reasons behind the relationship between academic research and social needs. And, as always, the balance of research effort between global and local needs for different countries also needs further exploration.

\section{List of abbreviations}

AIDs: acquired immunodeficiency syndrome; DALYs: disability-adjusted life years; GHE: Global Health Estimates; HBV: hepatitis B virus; HDI: Human Development Index; HIV: human immunodeficiency virus; ICD-10: International Classification of Diseases, Tenth Revision IPDs: infectious and parasitic diseases; NSI: normalized specialization index; PHEIC: Public Health Emergency of International Concerns; R\&D: research and development; SI: specialization index; UN: United Nations; UNDP: United Nations Development Programme; WHA: World Health Assembly; WHO: World Health Organization; WoS: Web of Science.

\section{Ethics approval and consent to participate}

Not applicable.

\section{Consent for publication}

Not applicable.

\section{Availability of data and materials}

The raw bibliometric data were collected from the Clarivate Analytics's Web of Science, which is a commercial database required to be accessed with a license. The raw data of disease burden and Human Development Index (HDI) were downloaded from the World Health Organization (WHO) and United Nations Development Programme (UNDP), respectively. Web addresses for acquiring disease burden data and HDI are provided in the "Data and methodology" section in the article. 


\section{Competing interests}

The authors declare that they have no competing interests.

\section{Funding}

This work is supported by National Natural Science Foundation of China (Grant Nos. 71573085, 71974150).

\section{Authors' contributions}

WZ, LZ and LW all contributed to the conception of the work. WZ and LZ participated in data screening, extraction and quality assessment. WZ analyzed the data and drafted the manuscript. LZ and LW

provided critical comments and contributed to the interpretation of analyzed results. All authors read and approved the final manuscript.

\section{Acknowledgements}

The authors would like to acknowledge support from the National Laboratory Center for Library and Information Science in Wuhan University.

\section{Authors' information}

Wenjing ZHAO: cady_zhao@whu.edu.cn

Lili Wang: wang@merit.unu.edu

Lin Zhang (Correspondence): linzhang1117@,whu.edu.cn 


\section{References}

1. Nii-Trebi NI. Emerging and neglected infectious diseases: Insights, advances, and challenges. BioMed Research International. 2017;2017:5245021.

2. Vos T, Lim SS, Abbafati C, Abbas KM, Abbasi M, Abbasifard M, et al. Global burden of 369 diseases and injuries in 204 countries and territories, 1990-2019: a systematic analysis for the Global Burden of Disease Study 2019. The Lancet. 2020;396(10258):1204-22.

3. Pallari E, Lewison G. How biomedical research can inform both clinicians and the general public. In: W. Glänzel, H. Moed, U. Schmoch, Thelwall M, editors. Springer handbook of science and technology indicators2019. p. 581607.

4. Zhang L, Zhao W, Sun B, Huang Y, Glänzel W. How scientific research reacts to international public health emergencies: a global analysis of response patterns. Scientometrics. 2020;124:747-73.

5. Fry CV, Cai X, Zhang Y, Wagner CS. Consolidation in a crisis: Patterns of international collaboration in early COVID-19 research. PLoS One. 2020;15(7):e0236307.

6. Lee JJ, Haupt JP. Scientific Collaboration on COVID-19 Amidst Geopolitical Tensions between the US and China. The Journal of Higher Education. 2020;92(2):303-29.

7. Cause-specific mortality, 2000-2019 [Internet]. 2020. Available from: https://www.who.int/data/gho/data/themes/mortality-and-global-health-estimates/ghe-leading-causes-of-death.

Accessed 15 June 2021

8. Peprah E, Wonkam A. Biomedical research, a tool to address the health issues that affect African populations. Global Health. 2013;9(50):1-10.

9. Human Development Index (HDI) [Internet]. 2020. Available from: http://hdr.undp.org/en/content/humandevelopment-index-hdi. Accessed 24 December 2020

10. The 2020 Human Development Report-The next frontier: human development and the anthropocene [Internet]. 2020. Available from: http://hdr.undp.org/sites/default/files/hdr2020.pdf. Accessed 14 June 2021

11. Sarewitz D, Pielke RA. The neglected heart of science policy: reconciling supply of and demand for science. Environmental Science \& Policy. 2007;10(1):5-16.

12. Hicks DJ, Stapleford TA. The virtues of scientific practice: MacIntyre, virtue ethics, and the historiography of science. Isis. 2016;107(3):449-72.

13. Wang L, Wang X, Piro F, Philipsen NJ. The effect of competitive public funding on scientific output: A comparison between China and the EU. Research Evaluation. 2020;rvaa023:1-13.

14. Anderson RS. Patrick Blackett in india: military consultant and scientific intervenor, 1947-72. Part two. Notes and Records of The Royal Society. 1999;53(3):345-59.

15. Ciarli T, Ràfols I. The relation between research priorities and societal demands: The case of rice. Research Policy. 2019;48(4):949-67.

16. Hessels LK, van Lente H, Smits R. In search of relevance: the changing contract between science and society. Science and Public Policy. 2009;36(5):387-401.

17. Zhang L, Zhao W, Liu J, Sivertsen G, Huang Y. Do national funding organizations properly address the diseases with the highest burden?: Observations from China and the UK. Scientometrics. 2020;125(2):1733-61. 
18. Wallace ML, Ràfols I. Institutional shaping of research priorities: a case study on avian influenza. Research Policy. 2018;47(10):1975-89.

19. Gläser J, G. L. Governing science: How science policy shapes research content. European Journal of sociology/Archives Européennes de sociologie. 2016;57(1):117-68.

20. Cassi L, Lahatte A, Rafols I, Sautier P, Turckheim Éd. Improving fitness: Mapping research priorities against societal needs on obesity. Journal of Informetrics. 2017;11(4):1095-113.

21. Gillum LA, Gouveia C, Dorsey ER, Pletcher. M, Mathers. C. D, McCulloch. C. E, et al. NIH disease funding levels and burden of disease. PLoS ONE. 2011;6(2):e16837.

22. Hsiehchen D, Espinoza M, Hsieh A. Disease burden and the advancement of biomedical knowledge. Scientometrics. 2016;110(1):321-33.

23. Overland I, K. SB. The misallocation of climate research funding. Energy Research \& Social Science. 2020;62:101349.

24. Begum M, Lewison G, Jassem J, Mixich V, Cufer T, Nurgozhin T, et al. Mapping cancer research across Central and Eastern Europe, the Russian Federation and Central Asia: Implications for future national cancer control planning. European Journal of Cancer. 2018;104:127-36.

25. Li A, Lewison G. Chinese Cancer Research in 2009-18 and the Disease Burden. Cancer Management and Research. 2020;12:5031-40.

26. Evans JA, Shim JM, Ioannidis JP. Attention to local health burden and the global disparity of health research. PLoS One. 2014;9(4):e90147.

27. Hagenaars N, de Kruif T, van de Laar L, Waltman L, Meijer I, Levi M, et al. The relationship between publication volume of biomedical research and burden of disease. 2019.

28. Nass SJ, Levit LA, Gostin LO, Institute of Medicine (US) Committee on Health Research and the Privacy of Health Information: The HIPAA Privacy Rule (Eds.). Beyond the HIPAA Privacy Rule: Enhancing privacy, improving health through research. 3. Washington (DC): National Academies Press (US); 2009.

29. Kalita A, Shinde S, Patel V. Public health research in India in the new millennium: a bibliometric analysis. Global Health Action. 2015;8:27576.

30. Confraria H, Wang L. Medical research versus disease burden in Africa. Research Policy. 2020;49(3):103916.

31. Kinge JM, Roxrud I, Vollset SE, Skirbekk V, Røttingen JA. Are the Norwegian health research investments in line with the disease burden? Health Research Policy and Systems. 2014;12(64):1-98.

32. Ramaswami R, Paulino E, Barrichello A, Nogueira-Rodrigues A, Bukowski A, St Louis J, et al. Disparities in breast, lung, and cervical cancer trials worldwide. Journal of Global Oncology. 2018;4:1-11.

33. Rochon PA, Mashari A, Cohen A, Misra A, Laxer D, Streiner DL, et al. Relation between randomized controlled trials published in leading general medical journals and the global burden of disease. Canadian Medical Association journal. 2004;170(11):1673-7.

34. Albarqouni L, Elessi K, Abu-Rmeileh NME. A comparison between health research output and burden of disease in Arab countries: evidence from Palestine. Health Research Policy and Systems. 2018;16(25):1-9.

35. Begum M, Lewison G, Wright JS, Pallari E, Sullivan R. European non-communicable respiratory disease 
research, 2002-13: Bibliometric study of outputs and funding. PloS ONE. 2016;11(4):e0154197.

36. Cuschieri S, Pallari E, Terzic N, Alkerw A, Sigurðardóttir AK. Mapping the burden of diabetes in five small countries in Europe and setting the agenda for health policy and strategic action. Health Research Policy and Systems. 2021;19(43):1-10.

37. Fonseca BP, Albuquerque PC, Zicker F. Neglected tropical diseases in Brazil: lack of correlation between disease burden, research funding and output. Tropical Medicine \& International Health 2020;25(11):1373-84.

38. Yegros-Yegros A, van de Klippe W, Abad-Garcia MF, Rafols I. Exploring why global health needs are unmet by research efforts: the potential influences of geography, industry and publication incentives. Health Research Policy and Systems. 2020;18(1):1-14.

39. Trivedi AN, Zaslavsky AM, Schneider EC, Ayanian JZ. Trends in the quality of care and racial disparities in Medicare managed care. The New England Journal of Medicine. 2005;353:692-700.

40. Ottersen T, Elovainio R, Evans DB. Toward a coherent global framework for health financing: recommendations and recent developments. Health Economics, Policy and Law. 2017;12(2):285-96.

41. Røttingen J-A, Regmi S, Eide M, Young AJ, Viergever RF, Årdal C, et al. Mapping of available health research and development data: what's there, what's missing, and what role is there for a global observatory? Lancet. 2013;382(9900):1286-307.

42. Tallon D, Chard J, Dieppe P. Relation between agendas of the research community and the research consumer. Lancet. 2000;355:2037-40.

43. Hanna M. Matching taxpayer funding to population health needs. Circulation research. 2015;116(8):1296-300.

44. Kozma C, Calero-Medina C. The role of South African researchers in intercontinental collaboration. Scientometrics. 2019;121(3):1293-321.

45. Zacca-González G, Chinchilla-Rodríguez Z, Vargas-Quesada B. Medical scientific output and specialization in Latin American countries. Scientometrics. 2018;115(3):1635-50.

46. WHO methods and data sources for global burden of disease estimates 2000-2015 [Internet]. Department of Information, Evidence and 2017. Available from: https://www.who.int/healthinfo/global burden_disease/GlobalDALYmethods_2000_2015.pdf. Accessed 2 March 2021

47. Persson O, Melin G, Danell R, Kaloudis A. Research collaboration at Nordic universities. Scientometrics. 1997;39:209-33.

48. Rafols I, Yegros A. Is research responding to health needs? SSRN Electronic Journal. 2017.

49. Human Development Index (HDI): Data center [Internet]. 2021. Available from: http://hdr.undp.org/en/content/human-development-index-hdi. Accessed 25 June 2021

50. GBD 2017 Cause of Death Collaborators. Global, regional, and national age-sex-specific mortality for 282 causes of death in 195 countries and territories, 1980-2017: a systematic analysis for the Global Burden of Disease Study 2017. Lancet. 2018;392(10159):1736-88.

51. Lewsion G. Have the European Union programmes made a difference to biomedical research outputs? 18th International Conference on Scientometrics and Informetrics; KU Leuven, Belgium2021. p. 651-62. 
52. Neglected tropical diseases - Summary [Internet]. 2020. Available from: https://www.who.int/neglected diseases/diseases/summary/en/. Accessed 02 March 2020

53. Barry MA, Murray KO, Hotez PJ, Jones KM. Impact of vectorborne parasitic neglected tropical diseases on child health. Archives of Disease in Childhood. 2016;101(7):640-7.

54. HIV/AIDS-Key facts [Internet]. 2020. Available from: https://www.who.int/news-room/fact-sheets/detail/hivaids. Accessed 15 July 2021

55. Smith J, Ahmed K, Whiteside A. Why HIV/AIDS should be treated as exceptional: arguments from sub-Saharan Africa and Eastern Europe. African Journal of AIDS Research : AJAR. 2011;10 Suppl 1:345-56.

56. HIV and Opportunistic Infections, Coinfections, and Conditions: HIV and Tuberculosis (TB) [Internet]. 2020. Available from: https://hivinfo.nih.gov/understanding-hiv/fact-sheets/hiv-and-tuberculosis-tb. Accessed 24 June 2021

57. Hepatitis [Internet]. 2020. Available from: https:/www.who.int/health-topics/hepatitis\#tab=tab 1. Accessed 13 July 2021

58. Hepatitis B Virus Infection - High Endemic Geographic Areas [Internet]. 2018. Available from: https://www.albertadoctors.org/Advocacy/Hepatitis_B_Endemic_Countries.pdf. Accessed 15 July 2021

59. Liu J, Fan DM. Hepatitis B in China. Lancet. 2007;369(9573):1582-3.

60. Diarrhoeal disease [Internet]. 2017. Available from: https://www.who.int/news-room/factsheets/detail/diarrhoeal-disease. Accessed 22 June 2021

61. More than 140,000 die from measles as cases surge worldwide [Internet]. 2019. Available from: https://www.who.int/news/item/05-12-2019-more-than-140-000-die-from-measles-as-cases-surge-worldwide.

Accessed 7 June 2021

62. Measles-Key facts [Internet]. 2019. Available from: https://www.who.int/news-room/fact-sheets/detail/measles. Accessed 5 June 2021

63. Leprosy (Hansen's disease) [Internet]. 2021. Available from: https://www.who.int/news-room/factsheets/detail/leprosy. Accessed 10 July 2021

64. NIAID Role in Neglected Tropical Diseases (NTD) Research [Internet]. 2015. Available from: https://www.niaid.nih.gov/research/niaid-role-neglected-tropical-diseases-ntd-research. Accessed 21 June 2021

65. Zarocostas J. How the Gates Foundation seeks to energise the global fight against neglected tropical diseases. The Pharmaceutical Journal. 2017;299(7903).

66. The Least Developed Countries Report 2020 [Internet]. 2020. Available from: https://unctad.org/system/files/official-document/ldcr2020_en.pdf. Accessed 10 July 2021

67. Parkhurst JO. The Ugandan success story? Evidence and claims of HIV-1 prevention. 2002;360(9326).

68. Genuis SJ, Genuis SK. HIV/AIDS prevention in Uganda: why has it worked. Postgraduate Medical Journal. 2005;81(960):615-7.

69. Death rate from HIV/AIDs [Internet]. 2017. Available from: https:/ourworldindata.org/grapher/hiv-deathrates?tab=table\&stackMode $=$ absolute\&time $=$ earliest. $.2017 \&$ country $=\sim$ UGA\&region=World. Accessed 23 July 2021 
70. Kakaire T, Schlech W, Coutinho A, Brough R, Parkes-Ratanshi R. The future of financing for HIV services in Uganda and the wider sub-Saharan Africa region: should we ask patients to contribute to the cost of their care? . BMC Public Health 2016; 16:896.

71. Dyer E. The Historiography of HIV and AIDS in Uganda. History in Africa, 35, 301-325. Retrieved July 31, 2021, from http://www.jstor.org/stable/25483725. Kampala, Uganda: Uganda AIDS Commission; 2004.

72. Salton G, Bergmark D. A citation study of computer science literature. IEEE Transactions on Professional Communication. 1979;PC-22(3):146-58.

73. Van Eck NJ, Waltman L. Software survey: VOSviewer, a computer program for bibliometric mapping. Scientometrics. 2010;84(2):523-38.

74. Murray CJL. Good practices for health statistics: lessons from the Millennium Development Goal health indicators. Lancet. 2007;369(9564):826-73.

75. Murray CJL, Frenk J. Health metrics and evaluation: strengthening the science. Lancet. 2008;371(9619):11911199.

76. Krone M, Dufner V, Wagner M, Gelbrich G, Ertl G, Heuschmann PU. Public funding for medical research in relation to the burden of disease caused by cardiovascular diseases and neoplasms in Germany. Clinical Research in Cardiology. 2018;107(9):737-44. 


\section{Appendices}

\section{A1. The search strategy on publications}

The publications on the 11 specific conditions in IPD and other categories are collected according to search terms built by Confraria and Wang (30). These terms were derived from the diseases listed in Global Health Estimates (GHE) cause categories established according to International Classification of Diseases, Tenth Revision (ICD-10) by WHO (46).

The GHE cause category has a hierarchical structure of 4 levels so that different levels of aggregation are included, as shown is Figure A1. Three broad cause groups and 23 sub-groups constitute Level 1 and level 2 respectively: Group I - communicable, maternal, perinatal and nutritional conditions (5 subgroups), Group II - noncommunicable diseases (16 sub-groups) and Group III - injuries (2 sub-groups). IPD is a Level-2 category under Group I, which is the largest category of communicable diseases and contains 11 specific conditions (Level 3).

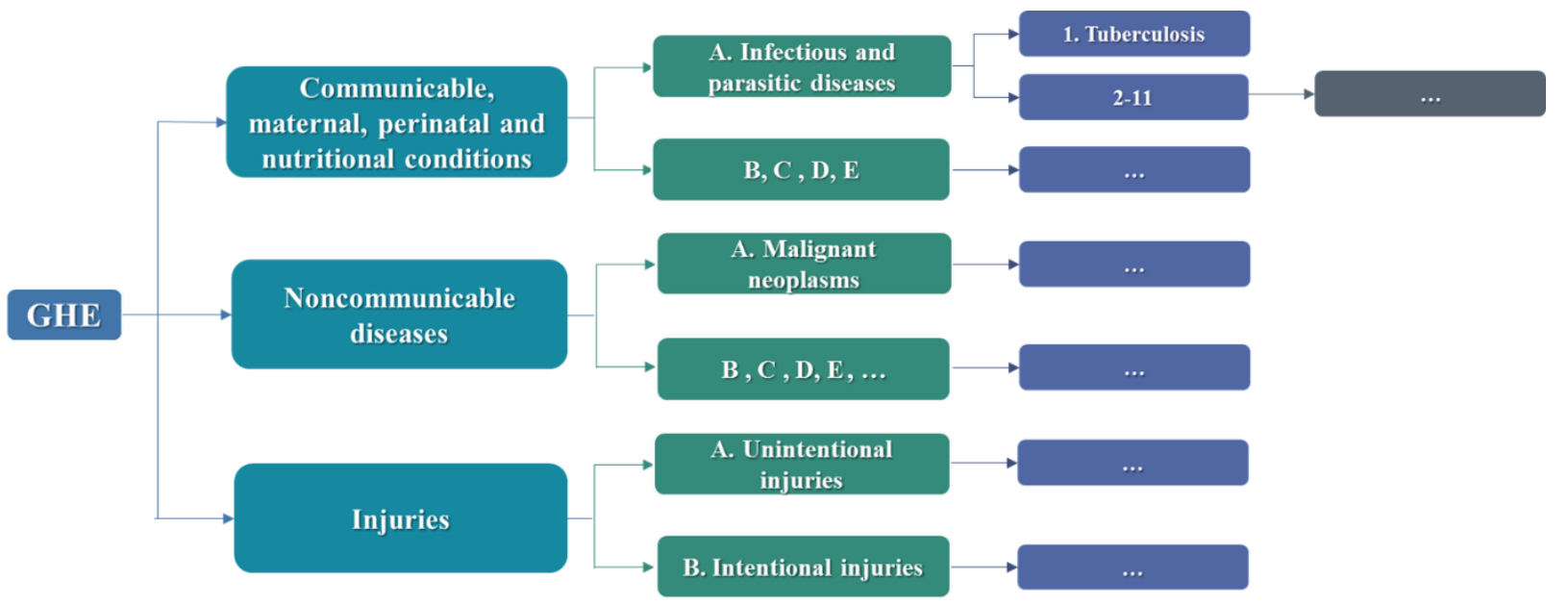

Figure A1. The hierarchical structure Global Health Estimates (GHE) cause category

As for the publication data retrieval, the search terms of 5 sub-groups in Group I and 13 sub-groups in Group II are included, in which the searching strategies of each 11 conditions (Level 3) in IPD are also provided in a more fine-grained approach by Confraria and Wang (30). The other sub-groups in Group II are exclude due to ambiguity. Further, because the Group III (injuries category) mainly refers to transportation and intentional/unintentional injuries rather than specific diseases, we did not include it in this study.

The search terms on each 11 specific conditions in IPD and diseases of other sub-groups are applied in "TI" (Title) and "AK" (Author Keywords) fields in advanced research in WoS to acquire publications (articles and reviews) on IPD and all the other diseases. Moreover, it should be noted that the terms of "all causes/diseases" used in the text refers to the sum of 5 sub-groups in Group I and 13 sub-groups in Group II. Correspondingly, "burden of all causes/diseases" also indicates the sum of the disease burdens in the above 18 sub-groups instead of that in the original 22 sub-groups in the cause category. 


\section{A2. The normalized publication specialization index ( NSI_Pub ) by human development level (all diseases)}

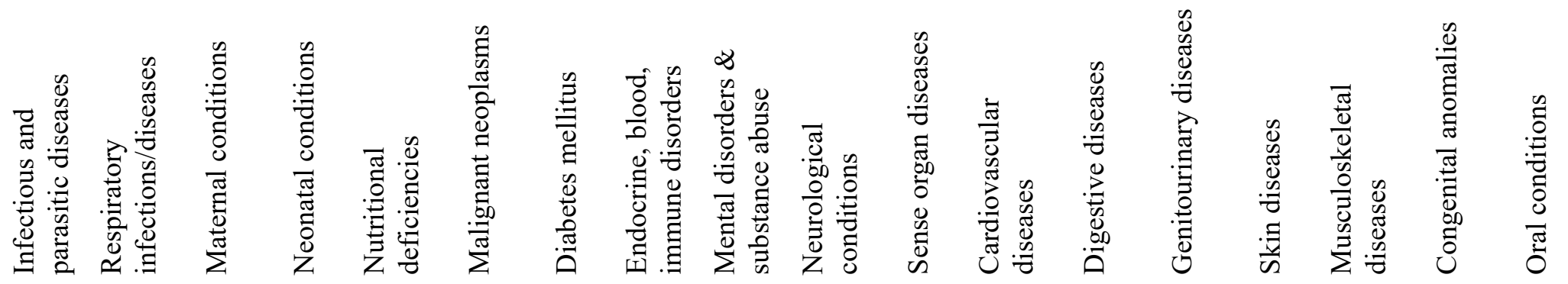

\begin{tabular}{|c|c|c|c|c|c|c|c|c|c|c|c|c|c|c|c|c|c|c|}
\hline \multicolumn{19}{|c|}{ 2000-04 } \\
\hline Low & 0.52 & -0.29 & 0.76 & -0.21 & 0.56 & -0.50 & -0.11 & -0.04 & -0.56 & -0.42 & -0.15 & -0.60 & -0.25 & -0.01 & -0.04 & -0.54 & -0.31 & 0.34 \\
\hline Med & 0.31 & -0.08 & 0.22 & 0.08 & 0.34 & -0.24 & -0.06 & -0.02 & -0.25 & -0.15 & -0.04 & -0.21 & -0.02 & 0.07 & -0.08 & -0.08 & -0.01 & 0.25 \\
\hline High & 0.11 & 0.00 & 0.10 & -0.19 & 0.05 & -0.08 & 0.12 & 0.04 & -0.21 & -0.02 & -0.15 & -0.02 & 0.01 & 0.09 & 0.01 & -0.03 & -0.07 & 0.00 \\
\hline Very high & -0.09 & 0.01 & -0.15 & 0.01 & -0.10 & 0.03 & 0.00 & 0.00 & 0.04 & 0.02 & 0.01 & 0.03 & 0.01 & -0.01 & 0.01 & 0.02 & 0.01 & -0.05 \\
\hline \multicolumn{19}{|c|}{ 2005-09 } \\
\hline Low & 0.51 & -0.20 & 0.70 & 0.03 & 0.50 & -0.41 & -0.03 & 0.02 & -0.51 & -0.32 & -0.09 & -0.50 & -0.16 & 0.01 & -0.06 & -0.42 & -0.13 & 0.24 \\
\hline Med & 0.21 & -0.02 & 0.05 & -0.03 & 0.21 & -0.09 & -0.02 & -0.06 & -0.25 & -0.14 & 0.05 & -0.12 & 0.06 & 0.10 & 0.09 & 0.02 & 0.05 & 0.08 \\
\hline High & 0.27 & 0.01 & 0.18 & -0.07 & 0.23 & -0.25 & 0.06 & 0.05 & -0.14 & -0.07 & -0.09 & -0.10 & -0.03 & 0.04 & -0.05 & -0.12 & -0.06 & 0.36 \\
\hline Very high & -0.11 & 0.01 & -0.15 & 0.01 & -0.10 & 0.04 & 0.00 & 0.00 & 0.04 & 0.03 & 0.00 & 0.03 & 0.00 & -0.02 & 0.00 & 0.02 & 0.00 & -0.07 \\
\hline \multicolumn{19}{|c|}{ 2010-14 } \\
\hline Low & 0.58 & -0.21 & 0.81 & 0.09 & 0.43 & -0.58 & -0.19 & 0.00 & -0.60 & -0.56 & -0.20 & -0.54 & -0.40 & -0.18 & -0.37 & -0.66 & -0.24 & 0.08 \\
\hline Med & 0.20 & -0.09 & 0.05 & -0.14 & 0.02 & 0.13 & 0.03 & -0.17 & -0.36 & -0.12 & -0.01 & -0.18 & 0.03 & 0.00 & -0.11 & -0.04 & -0.09 & 0.23 \\
\hline High & 0.20 & -0.02 & 0.05 & -0.07 & 0.17 & -0.22 & 0.03 & 0.05 & -0.17 & -0.10 & 0.00 & -0.10 & 0.01 & 0.11 & 0.12 & 0.00 & -0.02 & 0.28 \\
\hline Very high & -0.12 & 0.02 & -0.13 & 0.03 & -0.05 & 0.01 & -0.01 & 0.02 & 0.07 & 0.04 & 0.00 & 0.04 & 0.00 & -0.01 & 0.00 & 0.02 & 0.02 & -0.12 \\
\hline
\end{tabular}




\begin{tabular}{|c|c|c|c|c|c|c|c|c|c|c|c|c|c|c|c|c|c|c|}
\hline Low & 0.52 & -0.07 & 0.75 & 0.13 & 0.59 & -0.52 & 0.11 & 0.10 & -0.45 & -0.38 & -0.19 & -0.35 & -0.15 & -0.02 & -0.26 & -0.41 & -0.19 & 0.11 \\
\hline Med & 0.33 & -0.01 & 0.42 & 0.07 & 0.32 & -0.19 & 0.09 & 0.06 & -0.40 & -0.17 & 0.11 & -0.32 & 0.01 & 0.08 & 0.11 & -0.15 & 0.01 & 0.33 \\
\hline High & 0.10 & -0.03 & -0.18 & -0.11 & -0.03 & 0.11 & 0.04 & -0.16 & -0.23 & -0.08 & -0.07 & -0.09 & 0.02 & 0.01 & -0.10 & 0.04 & -0.14 & 0.16 \\
\hline Very high & -0.13 & 0.01 & -0.13 & 0.02 & -0.08 & -0.01 & -0.03 & 0.03 & 0.08 & 0.04 & 0.01 & 0.05 & 0.00 & -0.01 & 0.02 & 0.01 & 0.04 & -0.12 \\
\hline
\end{tabular}

